Document downloaded from:

http://hdl.handle.net/10251/126097

This paper must be cited as:

Calatayud-Gregori, J.; Cortés, J.; Jornet-Sanz, M. (2018). The damped pendulum random differential equation: A comprehensive stochastic analysis via the computation of the probability density function. Physica A Statistical Mechanics and its Applications. 512:261279. https://doi.org/10.1016/j.physa.2018.08.024

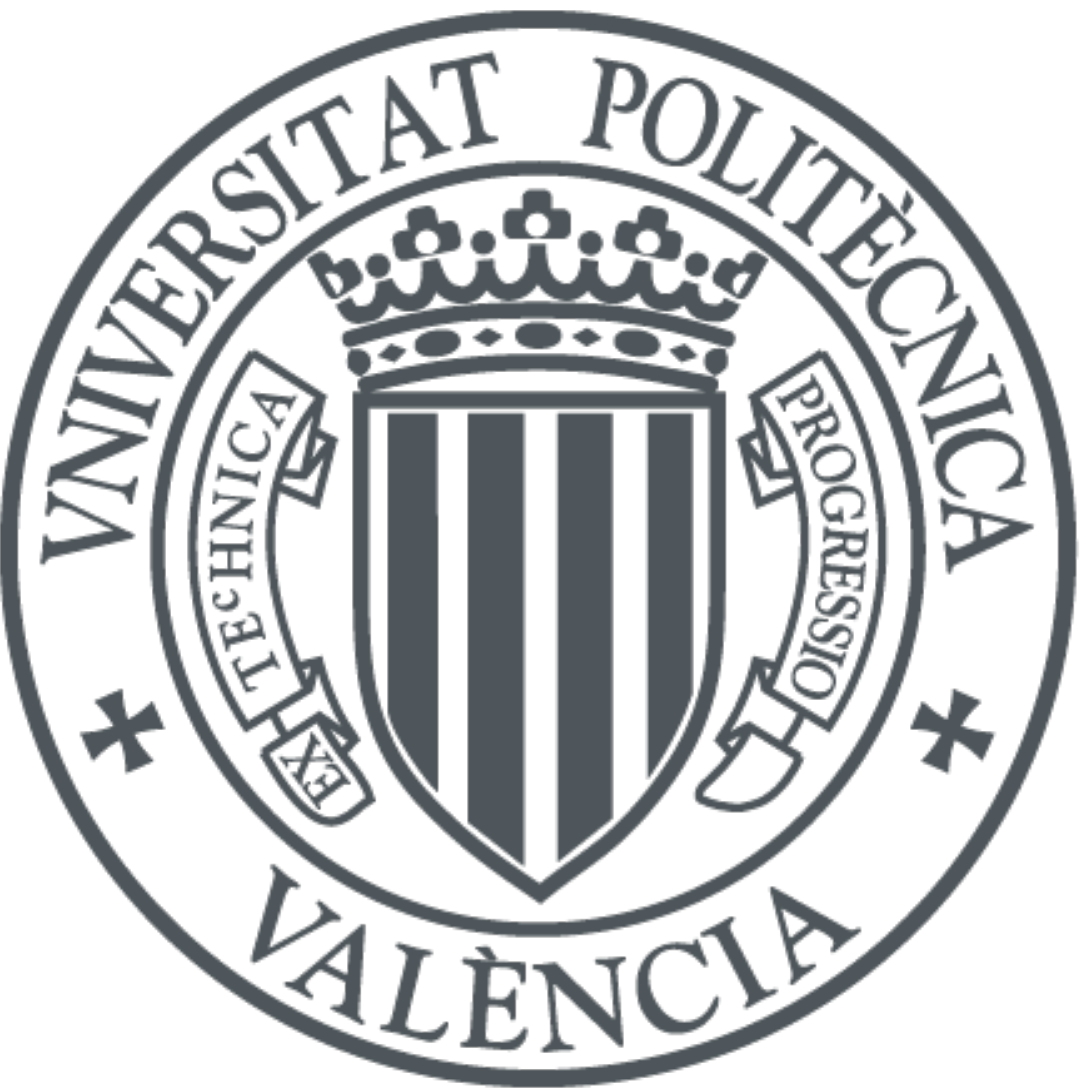

The final publication is available at

http://doi.org/10.1016/j.physa.2018.08.024

Copyright Elsevier

Additional Information 


\title{
THE DAMPED PENDULUM RANDOM DIFFERENTIAL EQUATION: A COMPREHENSIVE STOCHASTIC ANALYSIS VIA THE COMPUTATION OF THE PROBABILITY DENSITY FUNCTION
}

\author{
J. CALATAYUD, J.-C. CORTÉS, M. JORNET
}

\author{
Instituto Universitario de Matemática Multidisciplinar, \\ Universitat Politècnica de València, \\ Camino de Vera s/n, 46022, Valencia, Spain
}

email: jucagre@alumni.uv.es; jccortes@imm.upv.es; marjorsa@doctor.upv.es

\begin{abstract}
This paper deals with the damped pendulum random differential equation: $\ddot{X}(t)+2 \omega_{0} \xi \dot{X}(t)+\omega_{0}^{2} X(t)=Y(t), t \in[0, T]$, with initial conditions $X(0)=X_{0}$ and $\dot{X}(0)=X_{1}$. The forcing term $Y(t)$ is a stochastic process and $X_{0}$ and $X_{1}$ are random variables in a common underlying complete probability space $(\Omega, \mathcal{F}, \mathbb{P})$. The term $X(t)$ is a stochastic process that solves the random differential equation in both the sample path and in the $\mathrm{L}^{p}$ senses. To understand the probabilistic behaviour of $X(t)$, we need its joint finite-dimensional distributions. We establish mild conditions under which $X(t)$ is an absolutely continuous random variable, for each $t$, and we find its probability density function $f_{X(t)}(x)$. Thus, we obtain the first finite-dimensional distributions. In practice, we deal with two types of forcing term: $Y(t)$ is a Gaussian process, which occurs with the damped pendulum stochastic differential equation of Itô type; and $Y(t)$ can be approximated by a sequence $\left\{Y_{N}(t)\right\}_{N=1}^{\infty}$ in $\mathrm{L}^{2}([0, T] \times \Omega)$, which occurs with Karhunen-Loève expansions and some random power series. Finally, we provide numerical examples in which we choose specific random variables $X_{0}$ and $X_{1}$ and a specific stochastic process $Y(t)$, and then, we find the probability density function of $X(t)$.
\end{abstract}

Keywords: Damped pendulum random differential equation; Stochastic methods in Physics; Probability density function; Numerical analysis.

AMS Classification 2010: 34F05, 60H35, 65Z05, 60H10, 93E03.

\section{INTRODUCTION AND MOTIVATION}

The study of the damped pendulum differential equation with uncertainties has been tackled using different approaches, namely, the random and the Itô approaches, [1, pp. 96-97], [2]. In the former case, uncertainty is manifested in coefficients, initial/boundary conditions and/or forcing term via random variables and/or functions whose sample behaviour is regular (e.g., continuous). This approach leads to the socalled Random Differential Equations (RDEs). While in the latter case, uncertainty is forced through an irregular stochastic process such as a Wiener process or Brownian motion. In this case, Stochastic Differential Equations (SDEs) are formulated. 
SDEs are typically written in terms of stochastic differentials, but they are interpreted as Itô stochastic integrals. Nevertheless, if the integrand of the involved Itô integral is deterministic, SDEs can be treated as RDEs too. This is the case when the forcing term is just the White noise stochastic process. This particular situation will be studied later. Throughout our contribution RDEs will be considered only.

On the one hand, in [3, p. 161] the author analyzes the damped pendulum RDE when the forcing term is nonwhite and assuming that it is mean square continuous and wide-sense stationary with zero mean and having a given correlation function. On the other hand, recently in [4] the authors have provided an efficient computational method, based upon generalized hat basis functions, for solving stochastic Itô differential equations written in their integral equivalent form. In [4], a nice analysis of the stochastic pendulum problem is included. In both papers, a key objective is the computation of reliable approximations for the mean and the variance of the solution stochastic process. In [5], the author deals with the study of an oscillator subject to a random multiplicative noise with a spectral density (or power-spectrum) that decays as a power law at high frequencies. Although the computation of the first two statistical moments (mean and variance) of the solution is an important goal, in general, a more ambitious target is the computation of the exact or approximate probability distribution of the solution stochastic process to RDEs. In particular, a major challenge is to determine the probability density function of the solution, since from it one can obtain a full characterization of all one-dimensional statistical moments of the solution (hence including, just as particular cases, the mean and the variance). We point out that the computation of the probability density function of the solution stochastic process of some random ordinary and partial differential equations describing relevant problems in Physics and Engineering has been achieved, [6, 7, 8, 9, 10, 11, 12, 13, 14].

In this paper, we deal with the computation of the probability density function of the random initial value problem

$$
\left\{\begin{array}{l}
\ddot{X}(t)+2 \omega_{0} \xi \dot{X}(t)+\omega_{0}^{2} X(t)=Y(t), t \in[0, T], \\
X(0)=X_{0} \\
\dot{X}(0)=X_{1}
\end{array}\right.
$$

under mild conditions on the random input data $X_{0}, X_{1}$ and $Y(t)$. Here, $T>0$, $w_{0} \neq 0, \xi \neq 0$ and $\xi^{2}<1$ (underdamped case) are constant [3, Example 7.2]. We are assuming an underlying complete probability space $(\Omega, \mathcal{F}, \mathbb{P})$, where $\Omega$ is the sample space, $\mathcal{F}$ is a $\sigma$-algebra of events and $\mathbb{P}$ is a probability measure. The outcomes (i.e., the elements of $\Omega$ ) will be generically denoted by $\omega$. The initial position $X_{0}$ and the initial velocity $X_{1}$ are random variables and the source/forcing term $Y(t)$ is a stochastic process. The term $X(t)$ is a stochastic process that solves (1.1) in some probabilistic sense, see Theorem 1.2 . When we want to make the dependence on $\omega \in \Omega$ explicit, we will write $X_{0}(\omega), X_{1}(\omega), Y(t, \omega)$ and $X(t, \omega)$.

Notation 1.1. In this paper, we will work with Lebesgue spaces. Given a measure space $(S, \mathcal{A}, \mu)$, where $S$ is an abstract set, $\mathcal{A}$ is the $\sigma$-algebra and $\mu$ is the measure, we will use the notation $\mathrm{L}^{p}(S), 1 \leq p<\infty$, for the real-valued $p$ integrable measurable mappings in the Lebesgue sense: $f: S \rightarrow \mathbb{R}$ such that 
$\|f\|_{\mathrm{L}^{p}(S)}:=\left(\int_{S}|f|^{p} \mathrm{~d} \mu\right)^{1 / p}<\infty$. When $p=\infty$, the norm in $\mathrm{L}^{\infty}(S)$ is given by $\|f\|_{\mathrm{L}^{\infty}(S)}=\inf \{\sup \{|f(x)|: x \in S \backslash N\}: \mu(N)=0\}$ (this norm is the so-called "essential supremum"). In this paper, we will work with the particular cases of $S$ being a real interval $I$ with the real Lebesgue measure $\mathrm{d} \mu=\mathrm{d} x, S$ being a sample space $\Omega_{0}$ with a probability measure $\mu=\mathbb{Q}$ (we write $\mathrm{d} \mu=\mathrm{d} \mathbb{Q}=\mathbb{Q}(\mathrm{d} x)$, where $x \in \Omega_{0}$ is the variable of integration), and $S$ being a product space $I \times \Omega_{0}$ with the product measure $\mathrm{d} \mu=\mathrm{d} x \times \mathrm{d} \mathbb{Q}$. Notice that, in the latter case, the $p$-norm of a measurable map $f: I \times \Omega_{0} \rightarrow \mathbb{R}$ is given by $\|f\|_{\mathrm{L}^{p}\left(I \times \Omega_{0}\right)}=\left(\mathbb{E}\left[\int_{I}|f(x)|^{p} \mathrm{~d} x\right]\right)^{1 / p}$, where $\mathbb{E}$ stands for the expectation operator with respect to the probability $\mathbb{Q}$. The important case $p=2$ corresponds to the so-called mean square stochastic convergence that will be explicitly used in this paper. The shorten notation a.e. and a.s. will stand for "almost every" or "almost everywhere", and "almost surely", respectively.

One way to find a formal solution to 1.1 is by acting as in the deterministic case. The second-order linear differential equation is equivalent to the following first-order system of linear differential equations:

$$
\left(\begin{array}{l}
\dot{X}(t) \\
\ddot{X}(t)
\end{array}\right)=\left(\begin{array}{cc}
0 & 1 \\
-\omega_{0}^{2} & -2 \omega_{0} \xi
\end{array}\right)\left(\begin{array}{l}
X(t) \\
\dot{X}(t)
\end{array}\right)+\left(\begin{array}{c}
0 \\
Y(t)
\end{array}\right)
$$

Using the theory on deterministic first-order systems of linear differential equations, one obtains that a formal solution to (1.1) is given by

$$
X(t)=\left(\frac{\xi \mathrm{e}^{-\omega_{0} \xi t} \sin \left(\omega_{1} t\right)}{\sqrt{1-\xi^{2}}}+\mathrm{e}^{-\omega_{0} \xi t} \cos \left(\omega_{1} t\right)\right) X_{0}+\frac{\mathrm{e}^{-\omega_{0} \xi t} \sin \left(\omega_{1} t\right)}{\omega_{1}} X_{1}+\int_{0}^{t} h(t-s) Y(s) \mathrm{d} s,
$$

where

$$
\omega_{1}=\omega_{0} \sqrt{1-\xi^{2}} \neq 0, \quad h(t)=\frac{1}{\omega_{1}} \mathrm{e}^{-\xi \omega_{0} t} \sin \left(\omega_{1} t\right) .
$$

The goal of this contribution is to provide a comprehensive stochastic analysis of the random initial value problem $(1.1)$ via the exact or approximate computation of the probability density function of its solution stochastic process (1.3)-(1.4). Furthermore, for the sake of completeness, we first establish the following theorem where we show that the stochastic process $(1.3)-(1.4)$ is a rigorous solution to $(1.1)$ in the two main probabilistic senses usually considered in dealing with RDEs, namely the sample path sense and the $L^{p}$ sense (see for instance [3, Appendix A] and [3, Ch. 5-8], respectively).

Theorem 1.2. The following hold:

- Sample path solution: Suppose that the integral $\int_{0}^{t} h(t-s) Y(s) \mathrm{d} s$ in (1.3) is interpreted as a Lebesgue integral for each $\omega \in \Omega$ fixed (this is sometimes referred to as SP integral, see [15, Def. A-1]). If $Y$ has sample paths in $\mathrm{L}^{1}([0, T])$, then $X$ is the unique process with absolutely continuous sample paths that solve (1.1) for a.e. $t \in[0, T]$. If $Y$ has continuous sample paths, then $X$ is the unique process with $C^{1}([0, T])$ sample paths that solve (1.1) for every $t \in[0, T]$.

- $\mathrm{L}^{p}$-solution: Suppose that the integral $\int_{0}^{t} h(t-s) Y(s) \mathrm{d} s$ in 1 1.3) is interpreted as an $\mathrm{L}^{p}$-Riemann integral (see [3, p. 100]). If $Y$ is $\mathrm{L}^{p}$-continuous and $X_{0}, X_{1} \in \mathrm{L}^{p}(\Omega)$, then $X$ is the unique $\mathrm{L}^{p}$-solution [3, p. 118]. 
Proof. The first part is a consequence of the theory on deterministic differential equations (see Carathéodory theory in [16, pp. 28-30]). The second part follows from [3, Th. 7.1.1].

We will address the following important cases regarding the random nature of the forcing term $Y(t)$ : (1) it is Gaussian, and in particular, a White noise stochastic process; (2) it can be represented via a Karhunen-Loève expansion, and (3) it can be represented via a mean square random power series.

This paper is organized as follows. In Section 2, we first establish a key lemma that will be extensively used throughout the paper. This result allows us to give useful expressions for the probability density function, $f_{X(t)}(x)$, of the solution stochastic process $X(t)$ given by $(1.3)-(1.4)$ in terms of the expectation operator or explicitly. In Subsection 2.1, these expressions are also obtained in the significant case that the forcing term $Y(t)$ is Gaussian. This particular analysis includes the case in which $Y(t)$ is the White noise stochastic process that involves an Itô type SDE. Subsection 2.2 addresses the study of the RDE (1.1) in the case that the forcing term can be approximated by a stochastic process in $\mathrm{L}^{2}([0, T] \times \Omega)$. After providing general results in this setting, the analysis is divided into two important cases: (i) $Y(t)$ is expressed via a Karhunen-Loève expansion (see Subsubsection 2.2.1) and (ii) $Y(t)$ is expressed via a mean square random power series (see Subsubsection 2.2.2). Section 3 is devoted to illustrating our findings by means of a wide range of numerical examples. Finally, conclusions are drawn in Section 4.

\section{Probability Density function of the solution stochastic PRocess}

Our main goal in this paper is to establish conditions under which the solution stochastic process $X(t)$ given by (1.3)-1.4 is an absolutely continuous random variable for each $t \in[0, T]$, and then to compute its probability density function, $f_{X(t)}(x)$. Physically, the existence and computation of the probability density function of $X(t)$ means that the probability for the response to lie in a certain set $A$ at time $t$ can be calculated as $\mathbb{P}(X(t) \in A)=\int_{A} f_{X(t)}(x) \mathrm{d} x$. This allows computing the main statistical properties of the response process $X(t)$, say the mean, variance, or any specific moment.

For the purpose of determining the probability density function of $X(t)$, we need the following key lemma.

Lemma 2.1. [17, pp. 266-267] Let $Z_{1}$ and $Z_{2}$ be two independent real random variables defined in a common complete probability space $(\Omega, \mathcal{F}, \mathbb{P})$. Suppose that $Z_{1}$ is absolutely continuous. Then $Z_{1}+Z_{2}$ is absolutely continuous and has density function $f_{Z_{1}+Z_{2}}(u)=\mathbb{E}\left[f_{Z_{1}}\left(u-Z_{2}\right)\right]$.

In what follows, we will impose conditions on the initial position $X_{0}$ and the initial velocity $X_{1}$, see $\boldsymbol{H}_{\mathbf{0}}$ and $\boldsymbol{H}_{\mathbf{1}}$, so that $X(t)$ is an absolutely continuous random variable, for each $t$. As we shall see in the subsequent development, Lemma 2.1 will play a key role to obtain the probability density function of $X(t), f_{X(t)}(x)$.

Fix $t \in[0, T]$. We will assume one of the following two hypotheses: 
$H_{\mathbf{0}}: X_{0}$ is absolutely continuous, $\frac{\xi \sin \left(\omega_{1} t\right)}{\sqrt{1-\xi^{2}}}+\cos \left(\omega_{1} t\right) \neq 0, X_{0}$ and $\left(X_{1}, Y\right)$ are independent 1 .

$H_{1}$ : $X_{1}$ is absolutely continuous, $\sin \left(\omega_{1} t\right) \neq 0, X_{1}$ and $\left(X_{0}, Y\right)$ are independent.

Assume $\boldsymbol{H}_{\mathbf{0}}$. Then, using the Random Variable Transformation technique [18, Lemma 4.12],

$$
Z_{1}=\left(\frac{\xi \mathrm{e}^{-\omega_{0} \xi t} \sin \left(\omega_{1} t\right)}{\sqrt{1-\xi^{2}}}+\mathrm{e}^{-\omega_{0} \xi t} \cos \left(\omega_{1} t\right)\right) X_{0}
$$

is absolutely continuous, with density function given by

$$
f_{Z_{1}}(z)=f_{X_{0}}\left(\frac{z}{\frac{\xi \mathrm{e}^{-\omega_{0} \xi t} \sin \left(\omega_{1} t\right)}{\sqrt{1-\xi^{2}}}+\mathrm{e}^{-\omega_{0} \xi t} \cos \left(\omega_{1} t\right)}\right) \frac{1}{\left|\frac{\xi \mathrm{e}^{-\omega_{0} \xi t} \sin \left(\omega_{1} t\right)}{\sqrt{1-\xi^{2}}}+\mathrm{e}^{-\omega_{0} \xi t} \cos \left(\omega_{1} t\right)\right|} .
$$

Taking into account expression $(1.3)$ and applying Lemma 2.1 with $Z_{2}=\frac{\mathrm{e}^{-\omega_{0} \xi t} \sin \left(\omega_{1} t\right)}{\omega_{1}} X_{1}+$ $\int_{0}^{t} h(t-s) Y(s) \mathrm{d} s, X(t)$ is absolutely continuous and

$$
\begin{aligned}
& f_{X(t)}(x) \\
= & \mathbb{E}\left[f_{X_{0}}\left(\frac{x-\frac{\mathrm{e}^{-\omega_{0} \xi t} \sin \left(\omega_{1} t\right)}{\omega_{1}} X_{1}-\int_{0}^{t} h(t-s) Y(s) \mathrm{d} s}{\frac{\xi \mathrm{e}^{-\omega_{0} \xi t} \sin \left(\omega_{1} t\right)}{\sqrt{1-\xi^{2}}}+\mathrm{e}^{-\omega_{0} \xi t} \cos \left(\omega_{1} t\right)}\right)\right] \frac{1}{\left|\frac{\xi \mathrm{e}^{-\omega_{0} \xi t} \sin \left(\omega_{1} t\right)}{\sqrt{1-\xi^{2}}}+\mathrm{e}^{-\omega_{0} \xi t} \cos \left(\omega_{1} t\right)\right|} .
\end{aligned}
$$

Assume $\boldsymbol{H}_{\mathbf{1}}$. Proceeding as before, but now applying Lemma 2.1 with $Z_{1}=$ $\frac{\mathrm{e}^{-\omega_{0} \xi t} \sin \left(\omega_{1} t\right)}{\omega_{1}} X_{1}$ and $Z_{2}=\left(\frac{\xi \mathrm{e}^{-\omega_{0} \xi t} \sin \left(\omega_{1} t\right)}{\sqrt{1-\xi^{2}}}+\mathrm{e}^{-\omega_{0} \xi t} \cos \left(\omega_{1} t\right)\right) X_{0}+\int_{0}^{t} h(t-s) Y(s) \mathrm{d} s$, $X(t)$ is absolutely continuous with density function

$$
\begin{aligned}
& f_{X(t)}(x) \\
= & \mathbb{E}\left[f_{X_{1}}\left(\frac{x-\left(\frac{\xi \mathrm{e}^{-\omega_{0} \xi t} \sin \left(\omega_{1} t\right)}{\sqrt{1-\xi^{2}}}+\mathrm{e}^{-\omega_{0} \xi t} \cos \left(\omega_{1} t\right)\right) X_{0}-\int_{0}^{t} h(t-s) Y(s) \mathrm{d} s}{\frac{\mathrm{e}^{-\omega_{0} \xi t} \sin \left(\omega_{1} t\right)}{\omega_{1}}}\right)\right] \frac{1}{\frac{\mathrm{e}^{-\omega_{0} \xi t}\left|\sin \left(\omega_{1} t\right)\right|}{\left|\omega_{1}\right|}} .
\end{aligned}
$$

Summarizing, the following result has been established:

Proposition 2.2. The following statements hold:

- Assume $\boldsymbol{H}_{\mathbf{0}}$, then the probability density function of the solution stochastic process (1.3)-(1.4) to the random initial value problem (1.1) is given by (2.1).

- Assume $\boldsymbol{H}_{\mathbf{1}}$, then the probability density function of the solution stochastic process (1.3) -1.4) to the random initial value problem (1.1) is given by (2.2).

\footnotetext{
${ }^{1} X_{0}$ and $\left(X_{1}, Y\right)$ independent means that, for any points $t_{1}, \ldots, t_{m} \in[0, T], m \geq 1$, the random variable $X_{0}$ and the random vector $\left(X_{1}, Y\left(t_{1}\right), \ldots, Y\left(t_{m}\right)\right)$ are independent.
} 
In practice, the problem is to know the probability law of $I(t)=\int_{0}^{t} h(t-s) Y(s) \mathrm{d} s$. If we know the law $\mathbb{P}_{I(t)}=\mathbb{P} \circ I(t)^{-1}$ of $I(t)$, then (2.1) becomes

$$
\begin{aligned}
f_{X(t)}(x)= & \frac{1}{\left|\frac{\xi \mathrm{e}^{-\omega_{0} \xi t} \sin \left(\omega_{1} t\right)}{\sqrt{1-\xi^{2}}}+\mathrm{e}^{-\omega_{0} \xi t} \cos \left(\omega_{1} t\right)\right|} \\
& \cdot \int_{\mathbb{R}^{2}} f_{X_{0}}\left(\frac{x-\frac{\mathrm{e}^{-\omega_{0} \xi t} \sin \left(\omega_{1} t\right)}{\omega_{1}} x_{1}-y}{\frac{\xi \mathrm{e}^{-\omega_{0} \xi t} \sin \left(\omega_{1} t\right)}{\sqrt{1-\xi^{2}}}+\mathrm{e}^{-\omega_{0} \xi t} \cos \left(\omega_{1} t\right)}\right) \mathbb{P}_{\left(X_{1}, I(t)\right)}\left(\mathrm{d} x_{1}, \mathrm{~d} y\right),
\end{aligned}
$$

and $(2.2)$ becomes

$$
\begin{aligned}
& f_{X(t)}(x) \\
= & \frac{1}{\frac{\mathrm{e}^{-\omega_{0} \xi t}\left|\sin \left(\omega_{1} t\right)\right|}{\left|\omega_{1}\right|}} \int_{\mathbb{R}^{2}} f_{X_{1}}\left(\frac{x-\left(\frac{\xi \mathrm{e}^{-\omega_{0} \xi t} \sin \left(\omega_{1} t\right)}{\sqrt{1-\xi^{2}}}+\mathrm{e}^{-\omega_{0} \xi t} \cos \left(\omega_{1} t\right)\right) x_{0}-y}{\frac{\mathrm{e}^{-\omega_{0} \xi t} \sin \left(\omega_{1} t\right)}{\omega_{1}}}\right) \mathbb{P}_{\left(X_{0}, I(t)\right)}\left(\mathrm{d} x_{0}, \mathrm{~d} y\right) .
\end{aligned}
$$

As a consequence, both expressions for the probability density function $f_{X(t)}(x)$ can be computed. In fact, one does not need to compute those integrals analytically, since by sampling from $X_{0}, X_{1}$ and $I(t)$, we can approximate the expectations in (2.1) and (2.2) as accurate as desired by the Law of Large Numbers (Monte Carlo simulations).

Our objective in the following subsections is to analyze important cases in which (2.1) and (2.2) can be computed, or at least approximated, in practice. More specifically, the subsequent analysis will be split into two cases, depending on the random nature of $Y(t)$. First, in Subsection 2.1, we will deal with $Y(t)$ being a Gaussian stochastic process. This case will include the damped pendulum stochastic differential equation of Itô type in Subsubsection 2.1.1. Second, in Subsection 2.2, we will deal with a forcing term $Y(t)$ that can be approximated in $\mathrm{L}^{2}([0, T] \times \Omega)$. This second case will include two significant situations: $Y(t)$ is expressed as a Karhunen-Loève expansion, in Subsubsection 2.2.1, and $Y(t)$ is expressed as a random power series, in Subsubsection 2.2.2.

2.1. Gaussian forcing term. Suppose that $Y(t)$ is a Gaussian stochastic process. Then the process $I(t)=\left\{\int_{0}^{t} h(t-s) Y(s) \mathrm{d} s: t \in[0, T]\right\}$ is Gaussian. Indeed, if the integral is interpreted as a Lebesgue integral for each fixed $\omega \in \Omega$, then this fact follows from [19, Lemma 2.3]; if the integral is an $L^{p}$-Riemann integral, $p \geq 2$, then the Gaussianity follows from [3, Th. 4.6.4]. Moreover, its mean and variance are given by

$$
\mathbb{E}[I(t)]=\int_{0}^{t} h(t-s) \mathbb{E}[Y(s)] \mathrm{d} s=: \mu_{I}(t)
$$

and

$$
\mathbb{V}[I(t)]=\int_{0}^{t} \int_{0}^{t} h\left(t-s_{1}\right) h\left(t-s_{2}\right) \operatorname{Cov}\left[Y\left(s_{1}\right), Y\left(s_{2}\right)\right] \mathrm{d} s_{1} \mathrm{~d} s_{2}=: \sigma_{I}(t)^{2},
$$

respectively. Thus, $I(t) \sim \operatorname{Normal}\left(\mu_{I}(t), \sigma_{I}(t)^{2}\right)$. From this, if $\boldsymbol{H}_{\mathbf{0}}$ holds and, moreover, the random variable $X_{1}$ and the stochastic process $Y$ are independent (which 
implies the independence of $X_{1}$ and $I(t)$, i.e., $\left.\mathbb{P}_{\left(X_{1}, I(t)\right)}=\mathbb{P}_{X_{1}} \times \mathbb{P}_{I(t)}\right)$, we can compute (2.1) as

$$
\begin{aligned}
f_{X(t)}(x)= & \frac{1}{\left|\frac{\xi \mathrm{e}^{-\omega_{0} \xi t} \sin \left(\omega_{1} t\right)}{\sqrt{1-\xi^{2}}}+\mathrm{e}^{-\omega_{0} \xi t} \cos \left(\omega_{1} t\right)\right|} \\
& \cdot \int_{\mathbb{R}} \int_{\mathbb{R}} f_{X_{0}}\left(\frac{x-\frac{\mathrm{e}^{-\omega_{0} \xi t} \sin \left(\omega_{1} t\right)}{\omega_{1}}}{\frac{\xi \mathrm{e}^{-\omega_{0} \xi t} \sin \left(\omega_{1} t\right)}{\sqrt{1-\xi^{2}}}+\mathrm{e}^{-\omega_{0} \xi t} \cos \left(\omega_{1} t\right)}\right) f_{\operatorname{Normal}\left(\mu_{I}(t), \sigma_{I}(t)^{2}\right)}(y) \mathrm{d} y \mathbb{P}_{X_{1}}\left(\mathrm{~d} x_{1}\right) .
\end{aligned}
$$

If $\boldsymbol{H}_{\mathbf{1}}$ holds and, moreover, the random variable $X_{0}$ and the stochastic process $Y$ are independent, 2.2 is written as

$$
\begin{aligned}
& f_{X(t)}(x) \\
= & \frac{1}{\frac{\mathrm{e}^{-\omega_{0} \xi t}\left|\sin \left(\omega_{1} t\right)\right|}{\left|\omega_{1}\right|}} \int_{\mathbb{R}} \int_{\mathbb{R}} f_{X_{1}}\left(\frac{x-\left(\frac{\xi \mathrm{e}^{-\omega_{0} \xi t} \sin \left(\omega_{1} t\right)}{\sqrt{1-\xi^{2}}}+\mathrm{e}^{-\omega_{0} \xi t} \cos \left(\omega_{1} t\right)\right) x_{0}-y}{\frac{\mathrm{e}^{-\omega_{0} \xi t} \sin \left(\omega_{1} t\right)}{\omega_{1}}}\right) \\
& \cdot f_{\text {Normal }\left(\mu_{I}(t), \sigma_{I}(t)^{2}\right)}(y) \mathrm{d} y \mathbb{P}_{X_{0}}\left(\mathrm{~d} x_{0}\right) .
\end{aligned}
$$

On the other hand, bearing in mind expressions $(1.3)-(1.4)$ and Lemma 2.1, if $I(t) \sim \operatorname{Normal}\left(\mu_{I}(t), \sigma_{I}(t)^{2}\right)$ and $Y$ is independent of $\left(\overline{X_{0}}, X_{1}\right)$, but $\boldsymbol{H}_{\mathbf{0}}$ and $\boldsymbol{H}_{\mathbf{1}}$ do not hold, then $X(t)$ remains being absolutely continuous. By Lemma 2.1 with $Z_{1}=I(t)$ and $Z_{2}=\left(\frac{\xi \mathrm{e}^{-\omega_{0} \xi t} \sin \left(\omega_{1} t\right)}{\sqrt{1-\xi^{2}}}+\mathrm{e}^{-\omega_{0} \xi t} \cos \left(\omega_{1} t\right)\right) X_{0}+\frac{\mathrm{e}^{-\omega_{0} \xi t} \sin \left(\omega_{1} t\right)}{\omega_{1}} X_{1}$, the probability density function of $X(t)$ can be expressed as

$$
\begin{aligned}
& f_{X(t)}(x) \\
= & \mathbb{E}\left[f_{\operatorname{Normal}\left(\mu_{I}(t), \sigma_{I}(t)^{2}\right)}\left(x-\left(\frac{\xi \mathrm{e}^{-\omega_{0} \xi t} \sin \left(\omega_{1} t\right)}{\sqrt{1-\xi^{2}}}+\mathrm{e}^{-\omega_{0} \xi t} \cos \left(\omega_{1} t\right)\right) X_{0}-\frac{\mathrm{e}^{-\omega_{0} \xi t} \sin \left(\omega_{1} t\right)}{\omega_{1}} X_{1}\right)\right] \\
= & \int_{\mathbb{R}^{2}} f_{\operatorname{Normal}\left(\mu_{I}(t), \sigma_{I}(t)^{2}\right)}\left(x-\left(\frac{\xi \mathrm{e}^{-\omega_{0} \xi t} \sin \left(\omega_{1} t\right)}{\sqrt{1-\xi^{2}}}+\mathrm{e}^{-\omega_{0} \xi t} \cos \left(\omega_{1} t\right)\right) x_{0}-\frac{\mathrm{e}^{-\omega_{0} \xi t} \sin \left(\omega_{1} t\right)}{\omega_{1}} x_{1}\right) \\
& \cdot \mathbb{P}_{\left(X_{0}, X_{1}\right)}\left(\mathrm{d} x_{0}, \mathrm{~d} x_{1}\right) .
\end{aligned}
$$

Observe that, in order to derive expression (2.7), we have not needed that $X_{0}$ and $X_{1}$ be absolutely continuous random variables, respectively. This fact is a consequence of the key Lemma 2.1.

Below, we summarize the results previously established in the important case that the forcing term $Y=Y(t)$ is a Gaussian stochastic process:

Proposition 2.3. Let us consider the random initial value problem (1.1), where $Y=Y(t)$ is a Gaussian stochastic process. The following statements hold:

- Assume $\boldsymbol{H}_{\mathbf{0}}$ and that $X_{1}$ and $Y$ are independent, then the probability density function of the solution stochastic process (1.3)-(1.4) is given by (2.5).

- Assume $\boldsymbol{H}_{\mathbf{1}}$ and that $X_{0}$ and $Y$ are independent, then the probability density function of the solution stochastic process (1.3)-(1.4) is given by (2.6). 
- Assume that $Y$ and $\left(X_{0}, X_{1}\right)$ are independent, then the probability density function of the solution stochastic process (1.3) - (1.4) is given by (2.7).

2.1.1. Damped pendulum stochastic differential equation of Itô type. Let the forcing term $Y(t)$ be a White noise process: $Y$ is a Gaussian process, $\mathbb{E}[Y(t)]=0$ and $\mathbb{E}[Y(t) Y(s)]=\delta_{0}(t-s)$. In this case, using formulas $(2.3)$ and $(2.4), \mu_{I}(t)=0$ and $\sigma_{I}(t)^{2}=\int_{0}^{t} h(s)^{2} \mathrm{~d} s$, therefore $I(t) \sim \operatorname{Normal}\left(0, \int_{0}^{t} h(s)^{2} \mathrm{~d} s\right)$. Thus, if $Y$ is independent of $\left(X_{0}, X_{1}\right)$ and $\left(X_{0}, X_{1}\right)$ has any probability distribution, then the density function of the solution stochastic process $X(t)$ is given by (2.7). For the sake of completeness, we state this result in the following corollary:

Corollary 2.4. Let us consider the random initial value problem $(1.1)$, where $Y=$ $Y(t)$ is a White noise stochastic process. Assume that $Y$ and $\left(X_{0}, X_{1}\right)$ are independent. Then, the probability density function of the solution stochastic process (1.3) - (1.4) is given by (2.7)

Notice that, if $Y(t)$ is a White noise process, then the RDE problem (1.1) becomes a SDE of Itô type: if we denote

$$
Z(t)=\left(\begin{array}{c}
X(t) \\
\dot{X}(t)
\end{array}\right), \quad Z_{0}=\left(\begin{array}{l}
X_{0} \\
X_{1}
\end{array}\right), \quad A=\left(\begin{array}{cc}
0 & 1 \\
-\omega_{0}^{2} & -2 \omega_{0} \xi
\end{array}\right), \quad b=\left(\begin{array}{l}
0 \\
1
\end{array}\right),
$$

and $Y(t)=\dot{B}(t)$, where $B$ is a standard Brownian motion [18, Def. 5.11], then, from $(1.2)$, we obtain the following SDE of Itô type:

$$
\left\{\begin{array}{l}
\mathrm{d} Z(t)=A Z(t) \mathrm{d} t+b \mathrm{~d} B(t), t \in[0, T] \\
Z(0)=Z_{0}
\end{array}\right.
$$

The solution stochastic process $X(t)$ of $(1.1)$ corresponds to the first component of $Z(t)$ :

$$
X(t)=\left(\frac{\xi \mathrm{e}^{-\omega_{0} \xi t} \sin \left(\omega_{1} t\right)}{\sqrt{1-\xi^{2}}}+\mathrm{e}^{-\omega_{0} \xi t} \cos \left(\omega_{1} t\right)\right) X_{0}+\frac{\mathrm{e}^{-\omega_{0} \xi t} \sin \left(\omega_{1} t\right)}{\omega_{1}} X_{1}+\int_{0}^{t} h(t-s) \mathrm{d} B(s),
$$

where the integral is understood in the Itô sense.

2.2. Forcing term that can be approximated in $\mathrm{L}^{2}([0, T] \times \Omega)$. In this subsection, we assume that $Y \in \mathrm{L}^{2}([0, T] \times \Omega)$ and that there exists a sequence $\left\{Y_{N}\right\}_{N=1}^{\infty}$ of stochastic processes in $\mathrm{L}^{2}([0, T] \times \Omega)$ that converges to $Y$ in the topology of $\mathrm{L}^{2}([0, T] \times \Omega)$. This occurs, as we will see, with Karhunen-Loève expansions and some random power series. The first case will be studied in Subsubsection 2.2.1. whereas the second case will be analyzed in Subsubsection 2.2.2. Before considering these two important cases, in this subsection we will establish general results in order to determine computable expressions for the probability density function of the solution stochastic process $(1.3)-(1.4)$ to the random initial value problem (1.1) (see Theorem 2.5 and Theorem 2.7 later).

From the truncation $Y_{N}$ of the forcing term $Y$, we consider a truncation of the solution stochastic process 1.3 ):

$$
X_{N}(t)=\left(\frac{\xi \mathrm{e}^{-\omega_{0} \xi t} \sin \left(\omega_{1} t\right)}{\sqrt{1-\xi^{2}}}+\mathrm{e}^{-\omega_{0} \xi t} \cos \left(\omega_{1} t\right)\right) X_{0}+\frac{\mathrm{e}^{-\omega_{0} \xi t} \sin \left(\omega_{1} t\right)}{\omega_{1}} X_{1}+\int_{0}^{t} h(t-s) Y_{N}(s) \mathrm{d} s,
$$


where the integral is understood in the Lebesgue sense. Notice that $X_{N}(t) \rightarrow X(t)$ as $N \rightarrow \infty$ in $\mathrm{L}^{2}(\Omega)$, for each $t \in[0, T]$. Indeed, since $Y_{N} \rightarrow Y$ as $N \rightarrow \infty$ in $\mathrm{L}^{2}([0, T] \times \Omega)$ and $h$ is bounded on $[0, T]$ (see definition of $h$ in $(1.4)$ ), we have that $h(t-s) Y_{N}(s) \rightarrow h(t-s) Y(s)$ as $N \rightarrow \infty$ in $\mathrm{L}^{2}([0, t] \times \Omega, \mathrm{d} s \times \mathrm{d} \mathbb{P})$, for each $t \in[0, T]$, so by Cauchy-Schwarz inequality,

$$
\begin{aligned}
\mathbb{E}\left[\left|X_{N}(t)-X(t)\right|^{2}\right] & =\mathbb{E}\left[\left|\int_{0}^{t} h(t-s)\left(Y_{N}(s)-Y(s)\right) \mathrm{d} s\right|^{2}\right] \\
& \leq T\|h\|_{\mathrm{L}^{\infty}([0, T])}^{2}\left\|Y_{N}-Y\right\|_{\mathrm{L}^{2}([0, T] \times \Omega)}^{2} \stackrel{N \rightarrow \infty}{\longrightarrow} 0 .
\end{aligned}
$$

This shows that $X_{N}(t) \rightarrow X(t)$ as $N \rightarrow \infty$ in $\mathrm{L}^{2}(\Omega)$, for each $t \in[0, T]$. This is important, as the main statistical information of $X(t)$, say the expectation and variance, can be approximated by using the following key properties of mean square convergence: $\lim _{N \rightarrow \infty} \mathbb{E}\left[X_{N}(t)\right]=\mathbb{E}[X(t)]$ and $\lim _{N \rightarrow \infty} \mathbb{V}\left[X_{N}(t)\right]=\mathbb{V}[X(t)]$ (see [3, Th. 4.2.1, Th. 4.3.1]).

We will assume that $\boldsymbol{H}_{\mathbf{0}}$ entails that $X_{0}$ is independent of $\left(X_{1}, Y_{1}, \ldots, Y_{N}\right)$ for all $N \geq 1$ (analogously, $H_{\mathbf{1}}$ implies that $X_{1}$ is independent of $\left(X_{0}, Y_{1}, \ldots, Y_{N}\right)$ for all $N \geq 1$ ). Later on, in the particular cases of Karhunen-Loève expansions and random power series, we will prove this assumption in Remark 2.8 and Remark 2.12. respectively.

Under $\boldsymbol{H}_{\mathbf{0}}, X_{N}(t)$ is absolutely continuous with density function given by

$$
\begin{aligned}
f_{X_{N}(t)}(x)= & \mathbb{E}\left[f_{X_{0}}\left(\frac{x-\frac{\mathrm{e}^{-\omega_{0} \xi t} \sin \left(\omega_{1} t\right)}{\omega_{1}} X_{1}-\int_{0}^{t} h(t-s) Y_{N}(s) \mathrm{d} s}{\frac{\xi \mathrm{e}^{-\omega_{0} \xi t} \sin \left(\omega_{1} t\right)}{\sqrt{1-\xi^{2}}}+\mathrm{e}^{-\omega_{0} \xi t} \cos \left(\omega_{1} t\right)}\right)\right] \\
& \cdot \frac{1}{\left|\frac{\xi \mathrm{e}^{-\omega_{0} \xi t} \sin \left(\omega_{1} t\right)}{\sqrt{1-\xi^{2}}}+\mathrm{e}^{-\omega_{0} \xi t} \cos \left(\omega_{1} t\right)\right|}
\end{aligned}
$$

Under $\boldsymbol{H}_{\mathbf{1}}, X_{N}(t)$ is absolutely continuous with density function given by

$$
\begin{aligned}
& f_{X_{N}(t)}(x) \\
= & \mathbb{E}\left[f_{X_{1}}\left(\frac{x-\left(\frac{\xi \mathrm{e}^{-\omega_{0} \xi t} \sin \left(\omega_{1} t\right)}{\sqrt{1-\xi^{2}}}+\mathrm{e}^{-\omega_{0} \xi t} \cos \left(\omega_{1} t\right)\right) X_{0}-\int_{0}^{t} h(t-s) Y_{N}(s) \mathrm{d} s}{\frac{\mathrm{e}^{-\omega_{0} \xi t} \sin \left(\omega_{1} t\right)}{\omega_{1}}}\right)\right] \frac{1}{\frac{\mathrm{e}^{-\omega_{0} \xi t\left|\sin \left(\omega_{1} t\right)\right|}}{\left|\omega_{1}\right|}} .
\end{aligned}
$$

Theorem 2.5. The following statements for the probability density function, $f_{X(t)}(x)$, of the solution stochastic process (1.3) -(1.4) to the random initial value problem (1.1) hold:

- Assume $\boldsymbol{H}_{\mathbf{0}}$. If $f_{X_{0}}$ is continuous on $\mathbb{R}$ and $f_{X_{0}}(x) \leq a+b x^{2}$, for certain $a, b>0$, then $\lim _{N \rightarrow \infty} f_{X_{N}(t)}(x)=f_{X(t)}(x)$ for all $x \in \mathbb{R}$, being $f_{X_{N}(t)}(x)$ and $f_{X(t)}(x)$ defined by (2.10) and (2.1), respectively.

- Assume $\boldsymbol{H}_{\mathbf{1}}$. If $f_{X_{1}}$ is continuous on $\mathbb{R}$ and $f_{X_{1}}(x) \leq a+b x^{2}$, for certain $a, b>0$, then $\lim _{N \rightarrow \infty} f_{X_{N}(t)}(x)=f_{X(t)}(x)$ for all $x \in \mathbb{R}$, being $f_{X_{N}(t)}(x)$ and $f_{X(t)}(x)$ defined by 2.11) and (2.2), respectively. 
Proof. We will prove the first part, as the second one is analogous. Thus, let us assume $\boldsymbol{H}_{\mathbf{0}}, f_{X_{0}}$ continuous on $\mathbb{R}$ and $f_{X_{0}}(x) \leq a+b x^{2}$.

Let

$$
U_{N}=\frac{x-\frac{\mathrm{e}^{-\omega_{0} \xi t} \sin \left(\omega_{1} t\right)}{\omega_{1}} X_{1}-\int_{0}^{t} h(t-s) Y_{N}(s) \mathrm{d} s}{\frac{\xi \mathrm{e}^{-\omega_{0} \xi t} \sin \left(\omega_{1} t\right)}{\sqrt{1-\xi^{2}}}+\mathrm{e}^{-\omega_{0} \xi t} \cos \left(\omega_{1} t\right)}
$$

and

$$
U=\frac{x-\frac{\mathrm{e}^{-\omega_{0} \xi t} \sin \left(\omega_{1} t\right)}{\omega_{1}} X_{1}-\int_{0}^{t} h(t-s) Y(s) \mathrm{d} s}{\frac{\xi \mathrm{e}^{-\omega_{0} \xi t} \sin \left(\omega_{1} t\right)}{\sqrt{1-\xi^{2}}}+\mathrm{e}^{-\omega_{0} \xi t} \cos \left(\omega_{1} t\right)} .
$$

Since $Y_{N} \rightarrow Y$ as $N \rightarrow \infty$ in $\mathrm{L}^{2}([0, T] \times \Omega)$, and by $(2.9)$, we derive that $U_{N} \rightarrow U$ as $N \rightarrow \infty$ in $\mathrm{L}^{2}(\Omega)$.

By 2.10), to conclude that $\lim _{N \rightarrow \infty} f_{X_{N}(t)}(x)=f_{X(t)}(x)$, it suffices to show $f_{X_{0}}\left(U_{N}\right) \rightarrow f_{X_{0}}(U)$ as $N \rightarrow \infty$ in $\mathrm{L}^{1}(\Omega)$ (because convergence in $\mathrm{L}^{1}(\Omega)$ implies convergence of expectations). This will follow if we show that, for every subsequence $\left\{U_{N_{k}}\right\}_{k=1}^{\infty}$ of $\left\{U_{N}\right\}_{N=1}^{\infty}$, there exists another subsequence $\left\{U_{N_{k_{l}}}\right\}_{l=1}^{\infty}$ such that $f_{X_{0}}\left(U_{N_{k_{l}}}\right) \rightarrow f_{X_{0}}(U)$ as $l \rightarrow \infty$ in $\mathrm{L}^{1}(\Omega)^{2}$. Thus, fix a subsequence $\left\{U_{N_{k}}\right\}_{k=1}^{\infty}$. Since $U_{N_{k}} \rightarrow U$ as $k \rightarrow \infty$ in $\mathrm{L}^{2}(\Omega)$, by [20, Th. 4.9] there exists a subsequence $\left\{U_{N_{k_{l}}}\right\}_{l=1}^{\infty}$ and a random variable $V \in \mathrm{L}^{2}(\Omega)$ such that $U_{N_{k_{l}}}(\omega) \rightarrow U(\omega)$ as $l \rightarrow \infty$ a.s. and $\left|U_{N_{k_{l}}}(\omega)\right| \leq V(\omega)$ a.s., for all $l \geq 1$. As $f_{X_{0}}$ is continuous on $\mathbb{R}, f_{X_{0}}\left(U_{N_{k_{l}}}(\omega)\right) \rightarrow$ $f_{X_{0}}(U(\omega))$ as $l \rightarrow \infty$ a.s. Now, $f_{X_{0}}\left(U_{N_{k_{l}}}(\omega)\right) \leq a+b U_{N_{k_{l}}}(\omega)^{2} \leq a+b V(\omega)^{2} \in \mathrm{L}^{1}(\Omega)$. By the Dominated Convergence Theorem [21, result 11.32, p. 321], $f_{X_{0}}\left(U_{N_{k_{l}}}\right) \rightarrow$ $f_{X_{0}}(U)$ as $l \rightarrow \infty$ in $\mathrm{L}^{1}(\Omega)$, as wanted.

Remark 2.6. The conditions $f_{X_{i}}, i=0,1$, continuous on $\mathbb{R}$ and $f_{X_{i}}(x) \leq a+b x^{2}$, are the usual hypotheses imposed so that the Nemytskii operator $V \mapsto f_{X_{i}}(V)$ is continuous from $\mathrm{L}^{2}(\Omega)$ to $\mathrm{L}^{1}(\Omega)$, [22, pp. 15-17], [23, pp. 154-163]. Essentially, these hypotheses are mathematical restrictions on the probabilistic features of the initial position and/or initial velocity that permit proving that the density functions of the truncations $X_{N}(t)$ tend to the density function of $X(t)$ as $N \rightarrow \infty$.

From a practical standpoint, most of the probability density functions, like Beta (with shape parameters $\alpha, \beta \geq 1$ ), Gaussian, Gamma (with shape parameter $\alpha \geq 1$ and rate parameter $\beta>0$ ), etc., are bounded, so it is enough to take the constant $a>0$ big enough in order that the condition $f_{X_{i}}(x) \leq a+b x^{2}$, for $i=0$ or $i=1$, holds. Nevertheless, in order to further enlarge the class of random variables for which our theoretical findings can be applied, below we generalize Theorem 2.5 by assuming that the probability density functions are almost everywhere continuous rather than continuous. Recall that a real function is said to be almost everywhere continuous if it is continuous except on a set of Lebesgue measure zero. For example,

\footnotetext{
${ }^{2}$ Let $\left\{a_{n}\right\}_{n=1}^{\infty}$ be a sequence and $a$ be an element in a topological space. If for every subsequence $\left\{a_{n_{k}}\right\}_{k=1}^{\infty}$, there exists a subsequence $\left\{a_{n_{k_{l}}}\right\}_{l=1}^{\infty}$ such that $a_{n_{k_{l}}} \rightarrow a$ as $l \rightarrow \infty$, then $a_{n} \rightarrow a$ as $n \rightarrow \infty$. Indeed, if $a_{n}$ does not tend to $a$, there exists a neighbourhood $A$ of $a$ and a subsequence $\left\{a_{n_{k}}\right\}_{k=1}^{\infty}$ such that $a_{n_{k}} \notin A$, for all $k \geq 1$. By hypothesis, there is a subsequence $\left\{a_{n_{k_{l}}}\right\}_{l=1}^{\infty}$ that converges to $a$. But this contradicts the fact that $a_{n_{k_{l}}} \notin A$, for each $l \geq 1$.
} 
the uniform, exponential, truncated Gaussian, etc. distributions possess almost everywhere continuous density functions.

In Section 3, the application of both Theorem 2.5 and Theorem 2.7 will be illustrated.

Theorem 2.7. The following statements for the probability density function, $f_{X(t)}(x)$, of the solution stochastic process (1.3) -(1.4) to the random initial value problem (1.1) hold:

- Assume $\boldsymbol{H}_{\mathbf{0}}$. Suppose that $f_{X_{0}}$ is a.e. continuous on $\mathbb{R}$ and $f_{X_{0}}(x) \leq a+$ $b x^{2}$ for a.e. $x \in \mathbb{R}$, for certain $a, b>0$. If, in addition, $\boldsymbol{H}_{\mathbf{1}}$ holds, then $\lim _{N \rightarrow \infty} f_{X_{N}(t)}(x)=f_{X(t)}(x)$ for all $x \in \mathbb{R}$, being $f_{X_{N}(t)}(x)$ and $f_{X}(x)$ defined by (2.10) and (2.1), respectively.

- Assume $\boldsymbol{H}_{\mathbf{1}}$. Suppose that $f_{X_{1}}$ is a.e. continuous on $\mathbb{R}$ and $f_{X_{1}}(x) \leq a+$ b $x^{2}$ for a.e. $x \in \mathbb{R}$, for certain $a, b>0$. If, in addition, $\boldsymbol{H}_{\mathbf{0}}$ holds, then $\lim _{N \rightarrow \infty} f_{X_{N}(t)}(x)=f_{X(t)}(x)$ for all $x \in \mathbb{R}$, being $f_{X_{N}(t)}(x)$ and $f_{X}(x)$ defined by (2.11) and (2.2), respectively.

Proof. We show the first part, as the second one is analogous. As in the proof of Theorem 2.5, consider $U_{N}$ and $U$ defined by (2.12) and (2.13), respectively. Recall that $U_{N} \rightarrow U$ as $N \rightarrow \infty$ in $\mathrm{L}^{2}(\Omega)$. Since $\boldsymbol{H}_{\mathbf{1}}$ holds, by Lemma $2.1 U$ is absolutely continuous. This, together with the fact that $f_{X_{0}}$ is a.e. continuous, implies that the probability that $U$ belongs to the discontinuity set of $f_{X_{0}}$ is 0 . Thereby, by the Continuous Mapping Theorem [24, p. 7, Th. 2.3], $f_{X_{0}}\left(U_{N_{k_{l}}}(\omega)\right) \rightarrow f_{X_{0}}(U(\omega))$ a.s., as $l \rightarrow \infty$. Therefore, the proof from Theorem 2.5 works.

2.2.1. Forcing term expressed as a Karhunen-Loève expansion. Let $Y \in \mathrm{L}^{2}([0, T] \times$ $\Omega$ ). Consider its covariance integral operator

$$
\mathcal{C}: \mathrm{L}^{2}([0, T]) \rightarrow \mathrm{L}^{2}([0, T]), \quad \mathcal{C} f(t)=\int_{0}^{T} \mathbb{C o v}[Y(t), Y(s)] f(s) \mathrm{d} s .
$$

This operator is linear, compact, self-adjoint and nonnegative-definite [18, Th. 1.68, Lemma 1.72, Lemma 1.77]. Let $J \in \mathbb{N} \cup\{\infty\}$ be the dimension of the image of $\mathcal{C}$. By Hilbert-Schmidt Theorem [18, Th. 1.73], the nonzero eigenvalues of $\mathcal{C}$, repeated according to their multiplicity, form a sequence $\left\{\nu_{j}\right\}_{j=1}^{J}$ (we will not assume any particular ordering of the sequence of nonzero eigenvalues). Moreover, the sequence $\left\{\phi_{j}\right\}_{j=1}^{\infty}$ of eigenfunctions of $\mathcal{C}$ is an orthonormal basis of $\mathrm{L}^{2}([0, T])$. Here, $\phi_{j}$ is associated to $\nu_{j}$, and if $j>J$, then $\phi_{j}$ is associated to 0 .

By Karhunen-Loève Theorem [18, Th. 5.28], the process $Y=\{Y(t): t \in[0, T]\}$ can be expressed as

$$
Y(t)=\mu_{Y}(t)+\sum_{j=1}^{J} \sqrt{\nu_{j}} \phi_{j}(t) \xi_{j},
$$

where $\mu_{Y}(t)=\mathbb{E}[Y(t)]$ and $\left\{\xi_{j}\right\}_{j=1}^{J}$ is a sequence of random variables with zero expectation, unit variance and pairwise uncorrelated. These random variables $\left\{\xi_{j}\right\}_{j=1}^{J}$ have a closed expression:

$$
\xi_{j}=\frac{1}{\sqrt{\nu_{j}}} \int_{0}^{T}\left(Y(s)-\mu_{Y}(s)\right) \phi_{j}(s) \mathrm{d} s, \quad j=1, \ldots, J .
$$


Moreover, if $Y=\{Y(t): t \in[0, T]\}$ is a Gaussian process, then $\left\{\xi_{j}\right\}_{j=1}^{J}$ are independent and Gaussian. If $J=\infty$, the series 2.15 converges in $\mathrm{L}^{2}([0, T] \times \Omega)$.

Notice that

$I(t)=\int_{0}^{t} h(t-s) Y(s) \mathrm{d} s=\int_{0}^{t} h(t-s) \mu_{Y}(s) \mathrm{d} s+\sum_{j=1}^{J} \sqrt{\nu_{j}}\left(\int_{0}^{t} h(t-s) \phi_{j}(s) \mathrm{d} s\right) \xi_{j}$,

where, if $J=\infty$, the series is understood in $\mathrm{L}^{2}([0, T] \times \Omega)$.

Remark 2.8. If $\boldsymbol{H}_{\mathbf{0}}$ holds, then the random inputs $X_{0}$ and $Y$ are independent, i.e., $X_{0}$ and $\left(Y\left(t_{1}\right), \ldots, Y\left(t_{m}\right)\right)$ are independent, for each $t_{1}, \ldots, t_{m} \in[0, T], m \geq 1$. By (2.16), $X_{0}$ and $\left(\xi_{1}, \ldots, \xi_{J}\right)$ are independent. Indeed, as it is proved in [19, Lemma 2.3],

$$
\xi_{j} \in \overline{\left\{\frac{1}{\sqrt{\nu_{j}}} \sum_{k=1}^{m} \lambda_{k}\left(Y\left(t_{k}\right)-\mu_{Y}\left(t_{k}\right)\right) \phi_{j}\left(t_{k}\right): \lambda_{k} \in \mathbb{R}, t_{k} \in[0,1], m \geq 1\right\}} \mathrm{L}^{\mathrm{L}^{2}(\Omega)}
$$

(the overline stands for the closure). Since $X_{0}$ is independent to each sum

$$
\frac{1}{\sqrt{\nu_{j}}} \sum_{k=1}^{m} \lambda_{k}\left(Y\left(t_{k}\right)-\mu_{Y}\left(t_{k}\right)\right) \phi_{j}\left(t_{k}\right)
$$

we derive that $\left(\xi_{1}, \ldots, \xi_{J}\right)$ is a limit in $\mathrm{L}^{2}\left(\Omega ; \mathbb{R}^{J}\right)$ of random vectors that are independent to $X_{0}$, which implies the independence of $X_{0}$ and $\left(\xi_{1}, \ldots, \xi_{J}\right)$.

An analogous result is satisfied if $\boldsymbol{H}_{\mathbf{1}}$ holds, instead of $\boldsymbol{H}_{\mathbf{0}}$. This concludes the remark.

Suppose that $J<\infty$. Assume that $\boldsymbol{H}_{\mathbf{0}}$ holds, that we know the probability law of the random vector $\left(\xi_{1}, \ldots, \xi_{J}\right)$ and that $X_{1}$ and $\left(\xi_{1}, \ldots, \xi_{J}\right)$ are independent. Then (2.1) becomes

$$
\begin{aligned}
& f_{X(t)}(x)=\frac{1}{\left|\frac{\xi \mathrm{e}^{-\omega_{0} \xi t} \sin \left(\omega_{1} t\right)}{\sqrt{1-\xi^{2}}}+\mathrm{e}^{-\omega_{0} \xi t} \cos \left(\omega_{1} t\right)\right|} \\
& \cdot \int_{\mathbb{R}^{J}} \int_{\mathbb{R}} f_{X_{0}}\left(\frac{x-\frac{\mathrm{e}^{-\omega_{0} \xi t} \sin \left(\omega_{1} t\right)}{\omega_{1}} x_{1}-\int_{0}^{t} h(t-s) \mu_{Y}(s) \mathrm{d} s-\sum_{j=1}^{J} \sqrt{\nu_{j}}\left(\int_{0}^{t} h(t-s) \phi_{j}(s) \mathrm{d} s\right) \xi_{j}}{\frac{\xi \mathrm{e}^{-\omega_{0} \xi t} \sin \left(\omega_{1} t\right)}{\sqrt{1-\xi^{2}}}+\mathrm{e}^{-\omega_{0} \xi t} \cos \left(\omega_{1} t\right)}\right) \\
& \cdot \mathbb{P}_{X_{1}}\left(\mathrm{~d} x_{1}\right) \mathbb{P}_{\left(\xi_{1}, \ldots, \xi_{J}\right)}\left(\mathrm{d} \xi_{1}, \ldots, \mathrm{d} \xi_{J}\right) .
\end{aligned}
$$

If $\boldsymbol{H}_{\mathbf{1}}$ holds, and $X_{0}$ and $\left(\xi_{1}, \ldots, \xi_{J}\right)$ are independent, then 2.2 becomes

$$
\begin{aligned}
& f_{X(t)}(x)=\frac{1}{\frac{\mathrm{e}^{-\omega_{0} \xi t}\left|\sin \left(\omega_{1} t\right)\right|}{\left|\omega 1^{\mid}\right|}} \\
& \cdot \int_{\mathbb{R}^{J}} \int_{\mathbb{R}} f_{X_{1}}\left(\frac{x-\left(\frac{\xi \mathrm{e}^{-\omega_{0} \xi t} \sin \left(\omega_{1} t\right)}{\sqrt{1-\xi^{2}}}+\mathrm{e}^{-\omega_{0} \xi t} \cos \left(\omega_{1} t\right)\right) x_{0}-\int_{0}^{t} h(t-s) \mu_{Y}(s) \mathrm{d} s-\sum_{j=1}^{J} \sqrt{\nu_{j}}\left(\int_{0}^{t} h(t-s) \phi_{j}(s) \mathrm{d} s\right) \xi_{j}}{\frac{\mathrm{e}^{-\omega_{0} \xi t} \sin \left(\omega_{1} t\right)}{\omega_{1}}}\right) \\
& \cdot \mathbb{P}_{X_{0}}\left(\mathrm{~d} x_{0}\right) \mathbb{P}_{\left(\xi_{1}, \ldots, \xi_{J}\right)}\left(\mathrm{d} \xi_{1}, \ldots, \mathrm{d} \xi_{J}\right) .
\end{aligned}
$$

Therefore, both densities are computable in practice. 
If $J=\infty$, for each $N \geq 1$ we may define the truncations

$$
Y_{N}(t)=\mu_{Y}(t)+\sum_{j=1}^{N} \sqrt{\nu_{j}} \phi_{j}(t) \xi_{j} .
$$

Thus, we have a sequence $\left\{Y_{N}\right\}_{N=1}^{\infty}$ that converges to $Y$ in $\mathrm{L}^{2}([0, T] \times \Omega)$, according to Karhunen-Loève Theorem [18, Th. 5.28]. By what we have just seen, the density function of the truncation $X_{N}$ given by (2.8) is computable, as in (2.18) and (2.19) by taking $J=N$. If the assumptions of Theorem 2.5 or Theorem 2.7 hold, then we can approximate $f_{X(t)}$ by using $\lim _{N \rightarrow \infty} f_{X_{N}(t)}(x)=f_{X(t)}(x)$.

In fact, a more general result than Theorem 2.7 can be established in this setting:

Theorem 2.9. The following statements for the probability density function, $f_{X(t)}(x)$, of the solution stochastic process (1.3) - 1.4) to the random initial value problem (1.1) hold:

- Assume $\boldsymbol{H}_{\mathbf{0}}$. Suppose that $f_{X_{0}}$ is a.e. continuous on $\mathbb{R}$ and $f_{X_{0}}(x) \leq a+b x^{2}$ for a.e. $x \in \mathbb{R}$, for certain $a, b>0$. If, in addition, $\boldsymbol{H}_{\mathbf{1}}$ holds or some $\xi_{j}$ is absolutely continuous, independent of $\left(X_{1}, \xi_{1}, \ldots, \xi_{j-1}, \xi_{j+1}, \ldots\right)$ and with $\int_{0}^{t} h(t-s) \phi_{j}(s) \mathrm{d} s \neq 0$, then $\lim _{N \rightarrow \infty} f_{X_{N}(t)}(x)=f_{X(t)}(x)$ for all $x \in \mathbb{R}$, being $f_{X_{N}(t)}(x)$ and $f_{X}(x)$ defined by (2.10) and (2.1), respectively.

- Assume $\boldsymbol{H}_{\mathbf{1}}$. Suppose that $f_{X_{1}}$ is a.e. continuous on $\mathbb{R}$ and $f_{X_{1}}(x) \leq a+b x^{2}$ for a.e. $x \in \mathbb{R}$, for certain $a, b>0$. If, in addition, $\boldsymbol{H}_{\mathbf{0}}$ holds or some $\xi_{j}$ is absolutely continuous, independent of $\left(X_{0}, \xi_{1}, \ldots, \xi_{j-1}, \xi_{j+1}, \ldots\right)$ and with $\int_{0}^{t} h(t-s) \phi_{j}(s) \mathrm{d} s \neq 0$, then $\lim _{N \rightarrow \infty} f_{X_{N}(t)}(x)=f_{X(t)}(x)$ for all $x \in \mathbb{R}$, being $f_{X_{N}(t)}(x)$ and $f_{X}(x)$ defined by 2.11) and (2.2), respectively.

Proof. We prove the first part, as the second one is analogous. As in the proof of Theorem 2.5, consider $U_{N}$ and $U$ defined by (2.12) and (2.13), respectively, where $Y_{N}$ is defined by (2.20). If some $\xi_{j}$ is absolutely continuous, independent of $\left(X_{1}, \xi_{1}, \ldots, \xi_{j-1}, \xi_{j+1}, \ldots\right)$ and with $\int_{0}^{t} h(t-s) \phi_{j}(s) \mathrm{d} s \neq 0$, by Lemma $2.1 U$ is absolutely continuous. Thereby, the proof of Theorem 2.7 is applicable.

To provide a full analysis, it is interesting to observe that, in this setting of Karhunen-Loève expansions, sometimes $\boldsymbol{H}_{\mathbf{0}}$ and $\boldsymbol{H}_{\mathbf{1}}$ do not need to be satisfied (so both $X_{0}$ and $X_{1}$ may not be absolutely continuous). Consider the hypothesis

$H_{2}: \xi_{1}$ is absolutely continuous, $\int_{0}^{t} h(t-s) \phi_{1}(s) \mathrm{d} s \neq 0, \xi_{1}$ and $\left(X_{0}, X_{1}, \xi_{2}, \ldots, \xi_{j}\right)$ are independent, for each $j, 2 \leq j \leq J]^{3}$ (if $\xi_{1}$ does not satisfy $\boldsymbol{H}_{\mathbf{2}}$ but another $\xi_{j}$ instead, $j \neq 1$, then we may reorder the eigenvalues and eigenfunctions so that $\xi_{1}$ becomes $\xi_{j}$ ).

The random variable

$$
Z_{1}=\sqrt{\nu_{1}}\left(\int_{0}^{t} h(t-s) \phi_{1}(s) \mathrm{d} s\right) \xi_{1}
$$

\footnotetext{
${ }^{3}$ If $J<\infty$, this independence is reduced to $\xi_{1}$ and $\left(X_{0}, X_{1}, \xi_{2}, \ldots, \xi_{J}\right)$ be independent.
} 
is absolutely continuous, with density function

$$
f_{Z_{1}}(z)=f_{\xi_{1}}\left(\frac{z}{\sqrt{\nu_{1}}\left(\int_{0}^{t} h(t-s) \phi_{1}(s) \mathrm{d} s\right)}\right) \frac{1}{\sqrt{\nu_{1}}\left|\int_{0}^{t} h(t-s) \phi_{1}(s) \mathrm{d} s\right|},
$$

as a consequence of the Random Variable Transformation technique. By (1.3), (2.17) and Lemma 2.1, the solution $X(t)$ is absolutely continuous, with density function given by

$$
\begin{aligned}
& f_{X(t)}(x)=\frac{1}{\sqrt{\nu_{1}}\left|\int_{0}^{t} h(t-s) \phi_{1}(s) \mathrm{d} s\right|} \\
& \cdot \mathbb{E}\left[f _ { \xi _ { 1 } } \left(\frac{z-\left(\frac{\xi \mathrm{e}^{-\omega_{0} \xi t} \sin \left(\omega_{1} t\right)}{\sqrt{1-\xi^{2}}}+\mathrm{e}^{-\omega_{0} \xi t} \cos \left(\omega_{1} t\right)\right) X_{0}-\frac{\mathrm{e}^{-\omega_{0} \xi t} \sin \left(\omega_{1} t\right)}{\omega_{1}} X_{1}}{\sqrt{\nu_{1}}\left(\int_{0}^{t} h(t-s) \phi_{1}(s) \mathrm{d} s\right)}\right.\right. \\
& \left.\left.+\frac{-\int_{0}^{t} h(t-s) \mu_{Y}(s) \mathrm{d} s-\sum_{j=2}^{J} \sqrt{\nu_{j}}\left(\int_{0}^{t} h(t-s) \phi_{j}(s) \mathrm{d} s\right) \xi_{j}}{\sqrt{\nu_{1}}\left(\int_{0}^{t} h(t-s) \phi_{1}(s) \mathrm{d} s\right)}\right)\right] .
\end{aligned}
$$

If $J<\infty$, we may compute this explicitly. Otherwise, if $J=\infty$, we need to consider the truncation $X_{N}(t)$ given by (2.8), where $Y_{N}$ is defined in 2.20). With analogous proofs to those of Theorem 2.5 and Theorem 2.7, one arrives at the following results:

Theorem 2.10. Assume $\boldsymbol{H}_{2}$. If $f_{\xi_{1}}$ is continuous on $\mathbb{R}$ and $f_{\xi_{1}}(x) \leq a+b x^{2}$, for certain $a, b>0$, then $\lim _{N \rightarrow \infty} f_{X_{N}(t)}(x)=f_{X(t)}(x)$ for all $x \in \mathbb{R}$, where $f_{X_{N}(t)}$ is given by 2.21) with $J=N$ and $f_{X(t)}(x)$ is given by (2.21) with $J=\infty$.

Theorem 2.11. Assume $\boldsymbol{H}_{2}$. Suppose that $f_{\xi_{1}}$ is a.e. continuous on $\mathbb{R}$ and $f_{\xi_{1}}(x) \leq$ $a+b x^{2}$ for a.e. $x \in \mathbb{R}$, for certain $a, b>0$. If, in addition, $\boldsymbol{H}_{\mathbf{0}}$ holds or $\boldsymbol{H}_{\mathbf{1}}$ holds or some other $\xi_{j}(j \neq 1)$ satisfies $\boldsymbol{H}_{\mathbf{2}}$, then $\lim _{N \rightarrow \infty} f_{X_{N}(t)}(x)=f_{X(t)}(x)$ for all $x \in \mathbb{R}$, where $f_{X_{N}(t)}$ is given by 2.21) with $J=N$ and $f_{X(t)}(x)$ is given by 2.21) with $J=\infty$.

2.2.2. Forcing term expressed as a random power series. Sometimes, one may work with a forcing term $Y(t)$ that is an analytic stochastic process in the mean square sense [3, p. 99]: $Y(t)=\sum_{n=0}^{\infty} Y_{n} t^{n}$, where the sum converges in $\mathrm{L}^{2}(\Omega)$, for each $t \in[0, T]$. We will assume, in addition, that the convergence of the series holds in $\mathrm{L}^{2}([0, T] \times \Omega)$. Thus, if we define the partial sums $\left\{Y_{N}(t)=\sum_{n=0}^{N} Y_{n} t^{n}\right\}_{N=0}^{\infty}$, then they converge to $Y$ in $\mathrm{L}^{2}([0, T] \times \Omega)$.

Remark 2.12. If $\boldsymbol{H}_{\mathbf{0}}$ holds, then $X_{0}$ and $Y$ are independent. Let us see that $X_{0}$ and $\left(Y_{0}, \ldots, Y_{N}\right)$ are independent, for $0 \leq N<\infty$.

We have $Y_{n}=Y^{(n)}(0) / n$ !, where $Y^{(n)}$ is the $n$-th mean square derivative of $Y(t)$ [3, Ch. 4]. Thus, if we prove that $X_{0}$ and

$$
\left(Y\left(t_{1}^{0}\right), \ldots, Y\left(t_{m_{0}}^{0}\right), Y^{\prime}\left(t_{1}^{1}\right), \ldots, Y^{\prime}\left(t_{m_{1}}^{1}\right), \ldots, Y^{(N)}\left(t_{1}^{N}\right), \ldots, Y^{(N)}\left(t_{m_{N}}^{N}\right)\right)
$$

are independent, for each $t_{1}^{0}, \ldots, t_{m_{0}}^{0}, t_{1}^{1}, \ldots, t_{m_{1}}^{1}, \ldots, t_{1}^{N}, \ldots, t_{m_{N}}^{N} \in[0, T], m_{0}, \ldots, m_{N} \geq$ 1 , then $X_{0}$ and $\left(Y_{0}, \ldots, Y_{N}\right)$ will be independent, as wanted.

We prove this assertion by induction on $N \geq 0$. For $N=0$, we already know that $X_{0}$ and $\left(Y\left(t_{1}^{0}\right), \ldots, Y\left(t_{m_{0}}^{0}\right)\right)$ are independent for each $t_{1}^{0}, \ldots, t_{m_{0}}^{0} \in[0, T], m_{0} \geq 1$, 
because of the independence of $X_{0}$ and $Y$. We assume the assertion true for $N-1$ and we prove it for $N$. Write

$$
\begin{aligned}
& \left(Y\left(t_{1}^{0}\right), \ldots, Y\left(t_{m_{0}}^{0}\right), \ldots, Y^{(N-1)}\left(t_{1}^{N-1}\right), \ldots, Y^{(N-1)}\left(t_{m_{N-1}}^{N-1}\right), Y^{(N)}\left(t_{1}^{N}\right), \ldots, Y^{(N)}\left(t_{m_{N}}^{N}\right)\right) \\
= & \lim _{h \rightarrow 0}\left(Y\left(t_{1}^{0}\right), \ldots, Y\left(t_{m_{0}}^{0}\right), \ldots, Y^{(N-1)}\left(t_{1}^{N-1}\right), \ldots, Y^{(N-1)}\left(t_{m_{N-1}-1}^{N-1}\right),\right. \\
& \left.\frac{Y^{(N-1)}\left(t_{1}^{N}+h\right)-Y^{(N-1)}\left(t_{1}^{N}\right)}{h}, \ldots, \frac{Y^{(N-1)}\left(t_{m_{N}}^{N}+h\right)-Y^{(N-1)}\left(t_{m_{N}}^{N}\right)}{h}\right),
\end{aligned}
$$

where the limit is understood in $\mathrm{L}^{2}\left(\Omega ; \mathbb{R}^{m_{0}+m_{1}+\ldots+m_{N}}\right)$. By induction, $X_{0}$ is independent of each random vector

$$
\begin{aligned}
& \left(Y\left(t_{1}^{0}\right), \ldots, Y\left(t_{m_{0}}^{0}\right), \ldots, Y^{(N-1)}\left(t_{1}^{N-1}\right), \ldots, Y^{(N-1)}\left(t_{m_{N-1}-1}^{N-1}\right),\right. \\
& \left.\frac{Y^{(N-1)}\left(t_{1}^{N}+h\right)-Y^{(N-1)}\left(t_{1}^{N}\right)}{h}, \ldots, \frac{Y^{(N-1)}\left(t_{m_{N}}^{N}+h\right)-Y^{(N-1)}\left(t_{m_{N}}^{N}\right)}{h}\right) .
\end{aligned}
$$

This implies that $X_{0}$ and

$$
\left(Y\left(t_{1}^{0}\right), \ldots, Y\left(t_{m_{0}}^{0}\right), Y^{\prime}\left(t_{1}^{1}\right), \ldots, Y^{\prime}\left(t_{m_{1}}^{1}\right), \ldots, Y^{(N)}\left(t_{1}^{N}\right), \ldots, Y^{(N)}\left(t_{m_{N}}^{N}\right)\right)
$$

are independent, as wanted.

An analogous result is satisfied if $\boldsymbol{H}_{\mathbf{1}}$ holds, instead of $\boldsymbol{H}_{\mathbf{0}}$. This concludes the remark.

If $\boldsymbol{H}_{\mathbf{0}}$ holds, we know the probability law of the random vector $\left(Y_{0}, \ldots, Y_{N}\right)$, and $X_{1}$ and $\left(Y_{0}, \ldots, Y_{N}\right)$ are independent, then the density function of $X_{N}(t)$ is computable: 2.10 becomes

$$
\begin{aligned}
& f_{X_{N}(t)}(x)=\mathbb{E}\left[f_{X_{0}}\left(\frac{x-\frac{\mathrm{e}^{-\omega_{0} \xi t} \sin \left(\omega_{1} t\right)}{\omega_{1}} X_{1}-\sum_{n=0}^{N} Y_{n} \int_{0}^{t} h(t-s) s^{n} \mathrm{~d} s}{\frac{\xi \mathrm{e}^{-\omega_{0} \xi t} \sin \left(\omega_{1} t\right)}{\sqrt{1-\xi^{2}}}+\mathrm{e}^{-\omega_{0} \xi t} \cos \left(\omega_{1} t\right)}\right)\right] \\
& \cdot \frac{1}{\left|\frac{\xi \mathrm{e}^{-\omega_{0} \xi t} \sin \left(\omega_{1} t\right)}{\sqrt{1-\xi^{2}}}+\mathrm{e}^{-\omega_{0} \xi t} \cos \left(\omega_{1} t\right)\right|} \\
& =\frac{1}{\left|\frac{\xi \mathrm{e}^{-\omega_{0} \xi t} \sin \left(\omega_{1} t\right)}{\sqrt{1-\xi^{2}}}+\mathrm{e}^{-\omega_{0} \xi t} \cos \left(\omega_{1} t\right)\right|} \\
& \cdot \int_{\mathbb{R}^{N+1}} \int_{\mathbb{R}} f_{X_{0}}\left(\frac{x-\frac{\mathrm{e}^{-\omega_{0} \xi t} \sin \left(\omega_{1} t\right)}{\omega_{1}} x_{1}-\sum_{n=0}^{N} y_{n} \int_{0}^{t} h(t-s) s^{n} \mathrm{~d} s}{\frac{\xi \mathrm{e}^{-\omega_{0} \xi t} \sin \left(\omega_{1} t\right)}{\sqrt{1-\xi^{2}}}+\mathrm{e}^{-\omega_{0} \xi t} \cos \left(\omega_{1} t\right)}\right) \\
& \cdot \mathbb{P}_{X_{1}}\left(\mathrm{~d} x_{1}\right) \mathbb{P}_{\left(Y_{0}, \ldots, Y_{N}\right)}\left(\mathrm{d} y_{0}, \ldots, \mathrm{d} y_{N}\right) .
\end{aligned}
$$


If $\boldsymbol{H}_{\mathbf{1}}$ holds, and $X_{0}$ and $\left(Y_{0}, \ldots, Y_{N}\right)$ are independent, (2.11) becomes

$$
\begin{aligned}
& f_{X_{N}(t)}(x) \\
= & \mathbb{E}\left[f_{X_{1}}\left(\frac{x-\left(\frac{\xi \mathrm{e}^{-\omega_{0} \xi t} \sin \left(\omega_{1} t\right)}{\sqrt{1-\xi^{2}}}+\mathrm{e}^{-\omega_{0} \xi t} \cos \left(\omega_{1} t\right)\right) X_{0}-\sum_{n=0}^{N} Y_{n} \int_{0}^{t} h(t-s) s^{n} \mathrm{~d} s}{\frac{\mathrm{e}^{-\omega_{0} \xi t} \sin \left(\omega_{1} t\right)}{\omega_{1}}}\right)\right] \frac{1}{\frac{\mathrm{e}^{-\omega_{0} \xi t \mid} \sin \left(\omega_{1} t\right) \mid}{\left|\omega_{1}\right|}} \\
= & \frac{1}{\mathrm{e}^{-\omega_{0} \xi t\left|\sin \left(\omega_{1} t\right)\right|}} \int_{\mathbb{R}^{N+1}} \int_{\mathbb{R}} f_{X_{1}}\left(\frac{x-\left(\frac{\xi \mathrm{e}^{-\omega_{0} \xi t} \sin \left(\omega_{1} t\right)}{\sqrt{1-\xi^{2}}}+\mathrm{e}^{-\omega_{0} \xi t} \cos \left(\omega_{1} t\right)\right) x_{0}-\sum_{n=0}^{N} y_{n} \int_{0}^{t} h(t-s) s^{n} \mathrm{~d} s}{\frac{\mathrm{e}^{-\omega_{0} \xi t} \sin \left(\omega_{1} t\right)}{\omega_{1}}}\right) \\
& \cdot \mathbb{P}_{X_{0}}\left(\mathrm{~d} x_{0}\right) \mathbb{P}_{\left(Y_{0}, \ldots, Y_{N}\right)}\left(\mathrm{d} y_{0}, \ldots, \mathrm{d} y_{N}\right) .
\end{aligned}
$$

If the assumptions of Theorem 2.5 or Theorem 2.7 hold, then we can approximate $f_{X(t)}$ by using $\lim _{N \rightarrow \infty} f_{X_{N}(t)}(x)=f_{X(t)}(x)$.

In fact, a more general result than Theorem 2.7 can be proved in this setting:

Theorem 2.13. The following statements hold:

- Assume $\boldsymbol{H}_{\mathbf{0}}$. Suppose that $f_{X_{0}}$ is a.e. continuous on $\mathbb{R}$ and $f_{X_{0}}(x) \leq a+b x^{2}$ for a.e. $x \in \mathbb{R}$, for certain $a, b>0$. If, in addition, $H_{\mathbf{1}}$ holds or some $Y_{n}$ is absolutely continuous, independent of $\left(X_{1}, Y_{0}, \ldots, Y_{n-1}, Y_{n+1}, \ldots\right)$ and with $\int_{0}^{t} h(t-s) s^{n} \mathrm{~d} s \neq 0$, then $\lim _{N \rightarrow \infty} f_{X_{N}(t)}(x)=f_{X(t)}(x)$ for all $x \in \mathbb{R}$, being $f_{X_{N}(t)}(x)$ and $f_{X}(x)$ defined by (2.10) and (2.1), respectively.

- Assume $H_{1}$. Suppose that $f_{X_{1}}$ is a.e. continuous on $\mathbb{R}$ and $f_{X_{1}}(x) \leq a+b x^{2}$ for a.e. $x \in \mathbb{R}$, for certain $a, b>0$. If, in addition, $\boldsymbol{H}_{\mathbf{0}}$ holds or some $Y_{n}$ is absolutely continuous, independent of $\left(X_{0}, Y_{0}, \ldots, Y_{n-1}, Y_{n+1}, \ldots\right)$ and with $\int_{0}^{t} h(t-s) s^{n} \mathrm{~d} s \neq 0$, then $\lim _{N \rightarrow \infty} f_{X_{N}(t)}(x)=f_{X(t)}(x)$ for all $x \in \mathbb{R}$, being $f_{X_{N}(t)}(x)$ and $f_{X}(x)$ defined by (2.11) and (2.2), respectively.

Proof. Analogous to Theorem 2.9.

If $\boldsymbol{H}_{\mathbf{0}}$ and $\boldsymbol{H}_{\mathbf{1}}$ do not hold, we can consider the hypothesis

$H_{3}$ : There is an $n_{0} \geq 0$ such that $Y_{n_{0}}$ is absolutely continuous, $\int_{0}^{t} h(t-s) s^{n_{0}} \mathrm{~d} s \neq$ $0, Y_{n_{0}}$ and $\left(X_{0}, X_{1}, Y_{0}, \ldots, Y_{n_{0}-1}, Y_{n_{0}+1}, \ldots, Y_{N}\right)$ are independent, for each $N \geq 0$.

In such a case, the random variable $Z_{1}=Y_{n_{0}} \int_{0}^{t} h(t-s) s^{n_{0}} \mathrm{~d} s$ is absolutely continuous, with density function

$$
f_{Z_{1}}(z)=f_{Y_{n_{0}}}\left(\frac{z}{\int_{0}^{t} h(t-s) s^{n_{0}} \mathrm{~d} s}\right) \frac{1}{\left|\int_{0}^{t} h(t-s) s^{n_{0}} \mathrm{~d} s\right|} .
$$

By Lemma 2.1, for $N \geq n_{0}$,

$$
\begin{aligned}
& f_{X_{N}(t)}(x)=\frac{1}{\left|\int_{0}^{t} h(t-s) s^{n_{0}} \mathrm{~d} s\right|} \\
& \cdot \mathbb{E}\left[f_{Y_{n_{0}}}\left(\frac{x-\left(\frac{\xi \mathrm{e}^{-\omega_{0} \xi t} \sin \left(\omega_{1} t\right)}{\sqrt{1-\xi^{2}}}+\mathrm{e}^{-\omega_{0} \xi t} \cos \left(\omega_{1} t\right)\right) X_{0}-\frac{\mathrm{e}^{-\omega_{0} \xi t} \sin \left(\omega_{1} t\right)}{\omega_{1}} X_{1}-\sum_{n \neq n_{0}}^{N} Y_{n} \int_{0}^{t} h(t-s) s^{n} \mathrm{~d} s}{\int_{0}^{t} h(t-s) s^{n_{0}} \mathrm{~d} s}\right)\right] .
\end{aligned}
$$


With analogous proofs to those of Theorem 2.5 and Theorem 2.7, one arrives at the following results:

Theorem 2.14. Assume $\boldsymbol{H}_{\mathbf{3}}$. If $f_{Y_{n_{0}}}$ is continuous on $\mathbb{R}$ and $f_{Y_{n_{0}}}(x) \leq a+b x^{2}$, for certain $a, b>0$, then $\lim _{N \rightarrow \infty} f_{X_{N}(t)}(x)=f_{X(t)}(x)$ for all $x \in \mathbb{R}$, being $f_{X_{N}(t)}(x)$ given by 2.22) and $f_{X(t)}(x)$ given by 2.2. with $N=\infty$.

Theorem 2.15. Assume $\boldsymbol{H}_{\mathbf{3}}$. Suppose that $f_{Y_{n_{0}}}$ is a.e. continuous on $\mathbb{R}$ and $f_{Y_{n_{0}}}(x) \leq a+b x^{2}$ for a.e. $x \in \mathbb{R}$, for certain $a, b>0$. If, in addition, $\boldsymbol{H}_{\mathbf{0}}$ holds or $\boldsymbol{H}_{\mathbf{1}}$ holds or some other $Y_{n}, n \neq n_{0}$, satisfies $\boldsymbol{H}_{\mathbf{3}}$, then $\lim _{N \rightarrow \infty} f_{X_{N}(t)}(x)=f_{X(t)}(x)$ for all $x \in \mathbb{R}$, being $f_{X_{N}(t)}(x)$ given by (2.22) and $f_{X(t)}(x)$ given by (2.22) with $N=\infty$.

Remark 2.16. Hypotheses $\boldsymbol{H}_{\mathbf{0}}, \boldsymbol{H}_{\mathbf{1}}, \boldsymbol{H}_{\mathbf{2}}$ and $\boldsymbol{H}_{\mathbf{3}}$ are not necessary to have $X(t)$ absolutely continuous. Indeed, by [25], there exists a singular continuous measure $\lambda$ such that $\lambda * \lambda$ is absolutely continuous (here $*$ stands for the convolution operator). Fix $\bar{t} \in[0, T]$ such that

$$
\frac{\xi \sin \left(\omega_{1} \bar{t}\right)}{\sqrt{1-\xi^{2}}}+\cos \left(\omega_{1} \bar{t}\right) \neq 0, \quad \sin \left(\omega_{1} \bar{t}\right) \neq 0 .
$$

Take a pair of independent random variables $X_{0}$ and $X_{1}$ such that

$$
\left(\frac{\xi \mathrm{e}^{-\omega_{0} \xi \bar{t}} \sin \left(\omega_{1} \bar{t}\right)}{\sqrt{1-\xi^{2}}}+\mathrm{e}^{-\omega_{0} \xi \bar{t}} \cos \left(\omega_{1} \bar{t}\right)\right) X_{0} \sim \lambda, \quad \frac{\mathrm{e}^{-\omega_{0} \xi \bar{t}} \sin \left(\omega_{1} \bar{t}\right)}{\omega_{1}} X_{1} \sim \lambda .
$$

Let $Y=0$. Then the solution stochastic process $X(\bar{t})$ has distribution $\lambda * \lambda$, which is absolutely continuous. However, $\boldsymbol{H}_{\mathbf{0}}$ and $\boldsymbol{H}_{\mathbf{1}}$ do not hold, because $X_{0}$ and $X_{1}$ are not absolutely continuous, respectively. Thus, we have a pathological example. In these cases, at least to our knowledge, it is not possible to know the probability density function of the solution stochastic process.

\section{Applications}

In this section, we showcase the proposed approach on several examples where we apply our theoretical findings to particular random problems (1.1). The examples will cover a wide variety of situations which are of mathematical and physical interest. The main objective will be to test the methodology reported in this paper.

For the sake of clarity, throughout Examples 3.3 3.7 we will fix the constants in (1.1): we choose the upper time $T=1$, the damping ratio $\xi=1 / 2$ and the natural frequency $\omega_{0}=\pi / 2$. Then, according to (1.4), $\omega_{1}=\omega_{0} \sqrt{1-\xi^{2}}=\pi \sqrt{3} / 4$. Notice that, as $\omega_{1} \in(0, \pi / 2)$, we have $\sin \left(\omega_{1} t\right)>0$ and $\cos \left(\omega_{1} t\right)>0$, for $t \in(0,1]=(0, T]$. Then

$$
\frac{\xi \sin \left(\omega_{1} t\right)}{\sqrt{1-\xi^{2}}}+\cos \left(\omega_{1} t\right)>0
$$

on $[0,1]$ and $h(t)>0$ on $(0,1]$. We will work with independent $X_{0}, X_{1}$ and $Y$. Physically, the initial position, the initial velocity and the harmonic excitation are independent. On the other hand, to ensure the inequality $f(x) \leq a+b x^{2}$ for a specific density $f$, we will take $f$ bounded. Notice that this boundedness restriction covers any possible situation in practice, as a random variable with an unbounded density function may be truncated to achieve the boundedness condition but maintaining 
its main probabilistic features [26]. Moreover, truncation does not affect almost everywhere continuity of the density, which has been a very important hypothesis in some of the previous theorems.

Finally, to obtain/plot density functions given by an expectation expression ( 2.1$)$, (2.2), 2.7), 2.10), etc.), we will use Monte Carlo simulations.

Example 3.1. In this example we determine the probability density function of the response of a pendulum differential equation model to earthquake type random disturbances. Reference [27] justifies the use of equation (1.1) to model the response. Soong [3, Example 7.3] provides a summary of the results obtained in [27] and focuses on the mathematical properties of the random differential equation model, by computing the expectation and covariances, but not the probability density function. Reference [28] is a continuation of the investigation from [27]. On our part, our method to find the probability density function of the response will consist in a direct application of Lemma 2.1.

In [27, 28, [3, Example 7.3], a simple structure approximating a linear one-story building is considered. The goal is to analyze the response of this structure to an earthquake type random disturbance with ground acceleration

$$
Y(t)=\sum_{j=1}^{n} t a_{j} \mathrm{e}^{-\alpha_{j} t} \cos \left(w_{j} t+\theta_{j}\right), \quad t \geq 0 .
$$

It is assumed that $a_{j}, \alpha_{j}$ and $w_{j}$ are constant, while $\theta_{1}, \ldots, \theta_{n}$ are independent random variables with Uniform $(0,2 \pi)$ distribution. The term $Y(t)$ has this particular form because it takes the usual appearance of earthquake accelerogram records when $n$ is sufficiently large. Horizontal displacement of the roof of the structure is assumed due to ground motion. Let $X(t)$ be the relative horizontal displacement of the roof with respect to the ground (see [3] for further detailed physical justification). We assume that the roof is at rest at $t=0: X(0)=X_{0}=0$ and $\dot{X}(0)=X_{1}=0$. By [27], the relative displacement of the roof, $X(t)$, is governed by the pendulum differential equation

$$
\ddot{X}(t)+2 \omega_{0} \xi \dot{X}(t)+\omega_{0}^{2} X(t)=-Y(t) .
$$

The solution of this model is given by

$$
X(t)=-\int_{0}^{t} h(t-s) Y(s) \mathrm{d} s
$$

where the impulse response $h$ is given by (1.4).

In order to find the probability density function of $X(t)$, we rewrite $Y(t)$ as follows:

$$
Y(t)=t a_{1} \mathrm{e}^{-\alpha_{1} t}\left(\cos \left(w_{1} t\right) \cos \left(\theta_{1}\right)-\sin \left(w_{1} t\right) \sin \left(\theta_{1}\right)\right)+\sum_{j=2}^{n} t a_{j} \mathrm{e}^{-\alpha_{j} t} \cos \left(w_{j} t+\theta_{j}\right) .
$$

Then $X(t)$ can be written as $X(t)=Z_{1}+Z_{2}$, where

$$
Z_{1}=\left(-\int_{0}^{t} h(t-s) s a_{1} \mathrm{e}^{-\alpha_{1} s} \cos \left(w_{1} s\right) \mathrm{d} s\right) \cos \left(\theta_{1}\right)+\left(\int_{0}^{t} h(t-s) s a_{1} \mathrm{e}^{-\alpha_{1} s} \sin \left(w_{1} s\right) \mathrm{d} s\right) \sin \left(\theta_{1}\right),
$$




$$
Z_{2}=-\sum_{j=2}^{n} \int_{0}^{t} h(t-s) s a_{j} \mathrm{e}^{-\alpha_{j} s} \cos \left(w_{j} s+\theta_{j}\right) \mathrm{d} s .
$$

Since $\theta_{1}, \ldots, \theta_{n}$ are independent, for each fixed $t \geq 0$ the random variables $Z_{1}$ and $Z_{2}$ are also independent, and $Z_{1}$ is absolutely continuous, whose density function $f_{Z_{1}}$ can be obtained numerically by using that it is a transformation of $\theta_{1}$. By Lemma 2.1, the response $X(t)$ is absolutely continuous and its density function is given by $f_{X(t)}(x)=\mathbb{E}\left[f_{Z_{1}}\left(x-Z_{2}\right)\right]$. This expectation can be approximated by means of Monte Carlo simulations.

In order to obtain a graphical representation of the probability density function of $X(t)$, let us fix the following values for the constants: $\omega_{0}=20, \xi=0.05, n=20$, $\alpha_{i}=0.333, a_{i}=0.5$ and $w_{i}=15$, for $i=1, \ldots, 20$. In Figure 1 , we plot $f_{X(t)}(x)$ for $t=0.1$.

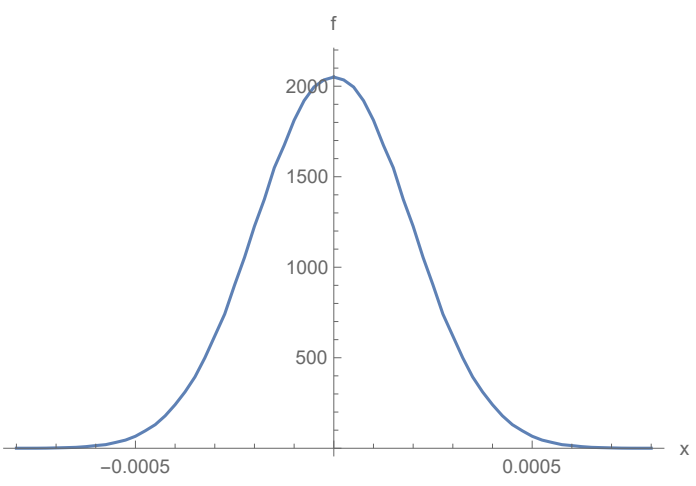

Figure 1. Density function $f_{X(t)}(x)$ for $t=0.1$ in Example 3.1 .

Example 3.2. White noise processes are of great interest in random vibrations. The probabilistic characteristics of dynamical systems with white noise inputs have been examined [29, 30, 31]. In this example, we consider the response of a massspring linear oscillator to a white noise random excitation, with governing equation $\ddot{X}(t)+\omega_{0}^{2} X(t)=Y(t)$, see [3, Example 7.1]. This is a particular case of 1.1) with damping ratio $\xi=0$. The source term $Y(t)$ is the formal derivative of a Brownian motion process, therefore the initial value problem (1.1) takes the form of a stochastic differential equation of Itô type. It is assumed that $X_{0}, X_{1}$ and $Y$ are independent.

As discussed in Subsection 2.1, the probability density function of the response $X(t)$ is expressed by 2.7 , being $\mu_{I}(t)=0$ and $\sigma_{I}(t)^{2}=\int_{0}^{t} h(s)^{2} \mathrm{~d} s$, where the impulse response $h$ is given by (1.4) .

For the numerical experiment, let us fix the frequency $\omega_{0}=1$, the initial position $X_{0}=0$ and the initial velocity $X_{1} \sim$ Triangular $(-0.1,0.1)$. In Figure 2, we depict the density function of $X(t)$ at $t=0.8$.

Example 3.3. Consider model (1.1) with fixed initial position $X_{0}=-1$, initial

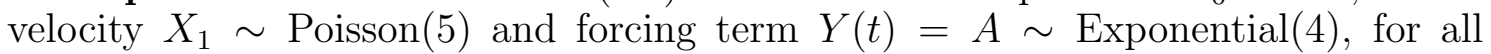
$t \in[0,1](Y(t)$ is a constant random variable as a function of $t$, i.e., a steadystate random excitation). These random variables are assumed to be independent. 


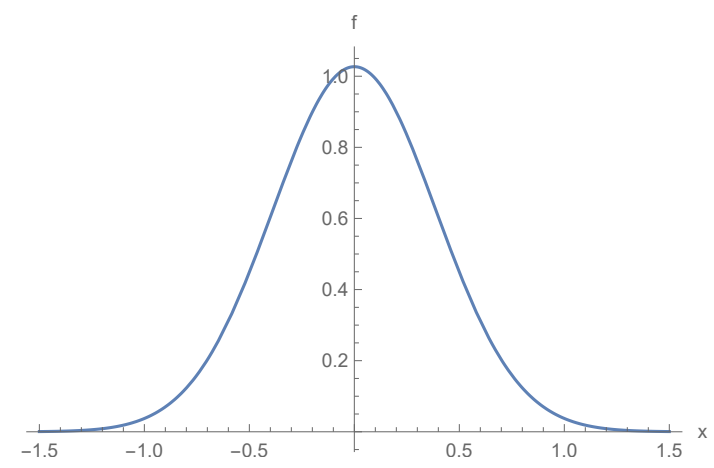

Figure 2. Density function $f_{X(t)}(x)$ for $t=0.8$ in Example 3.2

Using expression 2.22 with $N=n_{0}=0$ and $Y_{0}=A$, and taking into account that $\int_{0}^{t} h(t-s) s^{n_{0}} \mathrm{~d} s=\int_{0}^{t} h(t-s) \mathrm{d} s>0$, we deduce that the probability density function of the response stochastic process $X(t)$ is given by

$$
\begin{aligned}
& \frac{f_{X(t)}(x)}{\left|\int_{0}^{t} h(t-s) \mathrm{d} s\right|} \mathbb{E}\left[f_{A}\left(\frac{x-\left(\frac{\xi \mathrm{e}^{-\omega_{0} \xi t} \sin \left(\omega_{1} t\right)}{\sqrt{1-\xi^{2}}}+\mathrm{e}^{-\omega_{0} \xi t} \cos \left(\omega_{1} t\right)\right) X_{0}-\frac{\mathrm{e}^{-\omega_{0} \xi t} \sin \left(\omega_{1} t\right)}{\omega_{1}} X_{1}}{\int_{0}^{t} h(t-s) \mathrm{d} s}\right)\right],
\end{aligned}
$$

for $t \in(0,1]$. In Figure 3 , we show the graph of $f_{X(0.2)}(x)$, for $-1.5 \leq x \leq 1.5$.

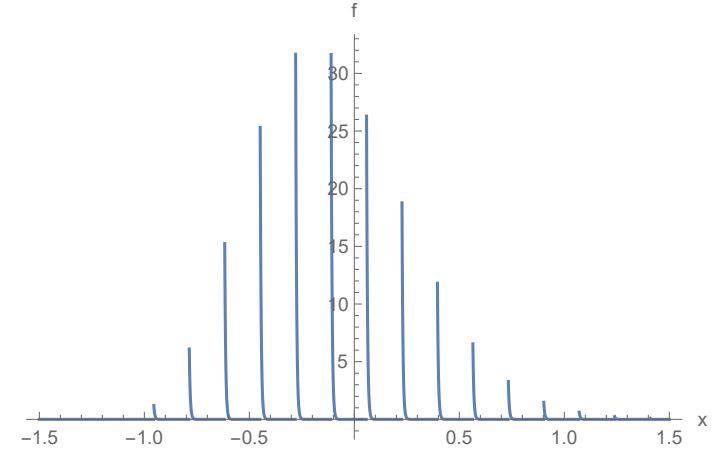

Figure 3. Density function $f_{X(0.2)}(x)$ in Example 3.3 .

Example 3.4. Let us consider model (1.1) with initial position $X_{0}$ having a Cantor distribution [32] and no initial motion, $X_{1}=0$. Recall that the Cantor distribution is defined by having as cumulative distribution function the Cantor staircase function. The Cantor distribution can be seen in the following way:

$$
X_{0}=2 \sum_{k=1}^{\infty} \frac{A_{k}}{3^{k}},
$$

where $A_{1}, A_{2}, \ldots$ are independent random variables with $\operatorname{Bernoulli}(0.5)$ distribution and the series converges a.s. Notice that $X_{0}$ is not an absolutely continuous random 
variable, hence hypothesis $\boldsymbol{H}_{\mathbf{0}}$ does not hold. We take the forcing term $Y(t)$ as a standard Brownian motion on [0,1] [18, Def. 5.11]. It is assumed that $X_{0}, X_{1}$ and $Y$ are independent.

Using formulas (2.3) and (2.4) and taking into account that $\mathbb{E}[Y(t)]=0$ and that $\operatorname{Cov}[Y(t), Y(s)]=\min \{t, s\}$, we compute $\mu_{I}(t)=0$ and $\sigma_{I}(t)^{2}=\int_{0}^{t} \int_{0}^{t} h\left(t-s_{1}\right) h(t-$ $\left.s_{2}\right) \min \left\{s_{1}, s_{2}\right\} \mathrm{d} s_{1} \mathrm{~d} s_{2}$.

According to Proposition 2.3, we have that the response $X(t)$ is absolutely continuous, with density function given by (2.7). In Figure 4, we have plotted the graph of $f_{X(0.1)}(x)$ and $f_{X(0.8)}(x)$. For $t=0.1$, we observe a big influence of the initial condition $X_{0}$. For $t=0.8$, the influence of $X_{0}$ seems to be dispelled, since $f_{X(0.8)}(x)$ is smoother.

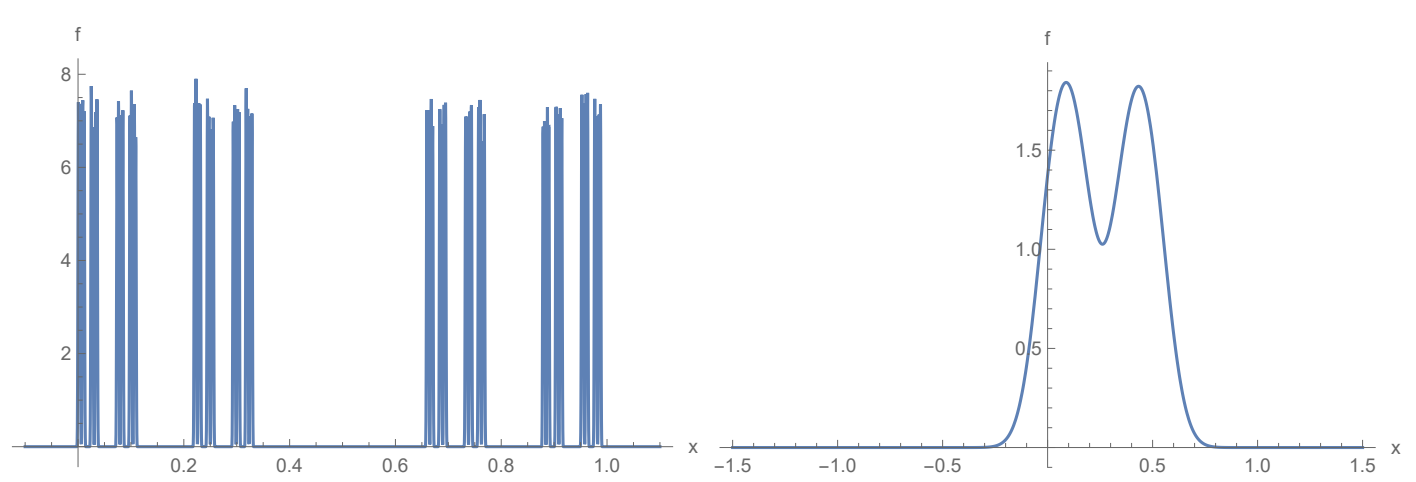

FIGURE 4. Left: Density function $f_{X(0.1)}(x)$. Right: Density function $f_{X(0.8)}(x)$. Both in the context of Example 3.4 and interpreting the Brownian motion $Y(t)=B(t)$ as a Gaussian stochastic process.

We may also see the Brownian motion as a Karhunen-Loève expansion [18, Exercise 5.12]:

$$
Y(t)=\sum_{j=1}^{\infty} \frac{\sqrt{2}}{\left(j-\frac{1}{2}\right) \pi} \sin \left(t\left(j-\frac{1}{2}\right) \pi\right) \xi_{j},
$$

where $\left\{\xi_{j}\right\}_{j=1}^{\infty}$ is a sequence of independent random variables with $\operatorname{Normal}(0,1)$ distribution. The series converges in $\mathrm{L}^{2}([0,1] \times \Omega)$.

By Theorem 2.10, the density function of $X_{N}(t)$ (given by (2.21) with $J=N$ ) converges pointwise to $f_{X(t)}(x)$, for $t \in(0,1]$ (given by $(2.21)$ with $J=\infty$ ). In Figure 5, we have plotted the graph of $f_{X_{15}(0.1)}(x)$ and $f_{X_{15}(0.8)}(x)(N=15)$. Notice that these densities approximate accurately the exact densities from Figure 4 , as Theorem 2.10 states.

Example 3.5. We deal with model (1.1) with initial position $X_{0} \sim$ Exponential(3), initial velocity $X_{1} \sim \operatorname{Binomial}(7,0.31)$ and forcing term

$$
Y(t)=\sum_{j=1}^{\infty} \frac{\sqrt{2}}{j \pi} \sin (t j \pi) \xi_{j} .
$$




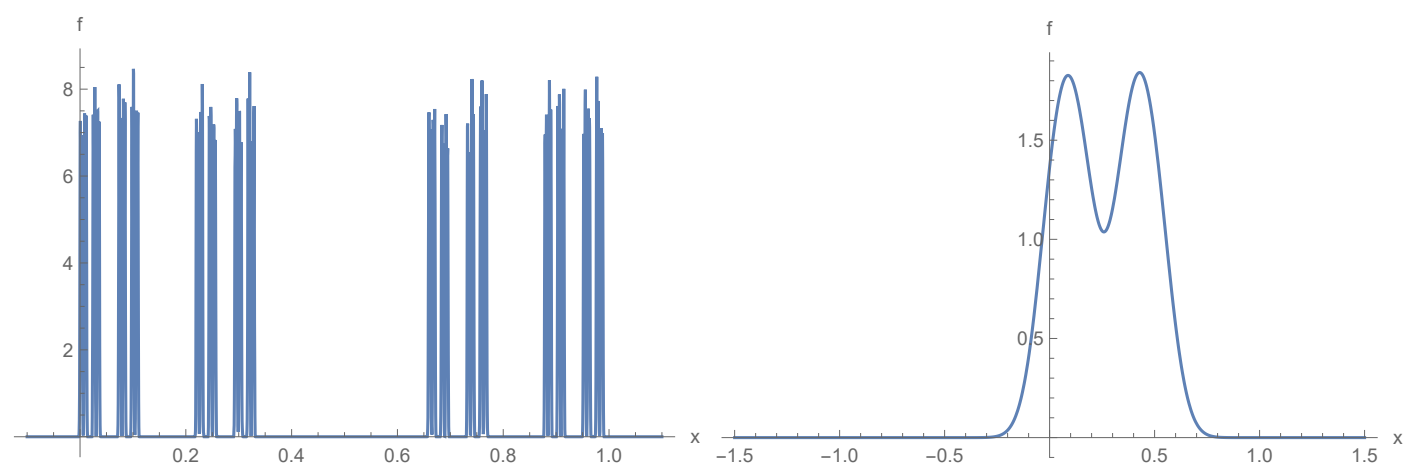

Figure 5. Left: Density function $f_{X_{15}(0.1)}(x)$. Right: Density function $f_{X_{15}(0.8)}(x)$. Both in the context of Example 3.4 and interpreting the Brownian motion $Y(t)=B(t)$ via its Karhunen-Loève expansion.

The series is understood in $\mathrm{L}^{2}([0,1] \times \Omega)$ and $\left\{\xi_{j}\right\}_{j=1}^{\infty}$ is a sequence of independent random variables with Uniform $(-\sqrt{3}, \sqrt{3})$ distribution. This is a Karhunen-Loève expansion. It is assumed that $X_{0}, X_{1}$ and $Y$ are independent.

Since $\phi_{1}(t)=\sin (t \pi)>0$ on $0<t<1$ and $h(t)>0$ on $0<t \leq 1$, then $\int_{0}^{t} h(t-s) \phi_{1}(s) \mathrm{d} s>0$, for $t \in(0,1]$. As a consequence, hypotheses $\boldsymbol{H}_{\mathbf{0}}$ and $\boldsymbol{H}_{\mathbf{2}}$ hold. By Theorem 2.9 or Theorem 2.11, $\lim _{N \rightarrow \infty} f_{X_{N}(t)}(x)=f_{X(t)}(x)$, for $t \in(0,1]$ and $x \in \mathbb{R}$.

In Figure 6 we have plotted $f_{X_{N}(0.5)}(x)$ for $N=1,2,3,4,5,6$. We observe convergence, since a small or nearly no variation of the density functions is noticed at different values of truncation order $N$. This convergence agrees with Theorem 2.9 and Theorem 2.11.

Example 3.6. Consider (1.1) with initial position $X_{0} \sim \operatorname{Gamma}(2,1)$, initial velocity $X_{1} \sim \operatorname{Beta}(1 / 2,1 / 2)$ and forcing term

$$
Y(t)=\sum_{j=1}^{\infty} \frac{\sqrt{2}}{j \pi} \sin (t j \pi) \xi_{j},
$$

where the series is understood in $\mathrm{L}^{2}([0,1] \times \Omega)$ and $\left\{\xi_{j}\right\}_{j=1}^{\infty}$ is a sequence of independent random variables with Uniform $\{-1,1\}$ distribution (discrete distribution with $\left.\mathbb{P}\left(\xi_{j}=-1\right)=\mathbb{P}\left(\xi_{j}=1\right)=1 / 2\right)$. This is a Karhunen-Loève expansion. It is assumed that $X_{0}, X_{1}$ and $Y$ are independent.

By Theorem 2.5. $\lim _{N \rightarrow \infty} f_{X_{N}(t)}(x)=f_{X(t)}(x)$, for $t \in[0,1]$ and $x \in \mathbb{R}$. In Figure 7 we plot $f_{X_{N}(0.5)}(x)$ for $N=1,2,3,4,5,6$. Convergence seems to be achieved, since nearly no variation of the density functions is noticed at different values of truncation order $N$. This fact agrees with Theorem 2.5 .

Example 3.7. Consider the initial value problem (1.1) with initial position $X_{0} \sim$ Negative Binomial $(7,0.31)$ and initial velocity $X_{1}$ being absolutely continuous with density function given by $f_{X_{1}}(x)=\sqrt{2} /\left(\pi\left(1+x^{4}\right)\right), x \in \mathbb{R}$. Take

$$
Y(t)=\sum_{n=1}^{\infty} \frac{A_{n}}{n} t^{n},
$$




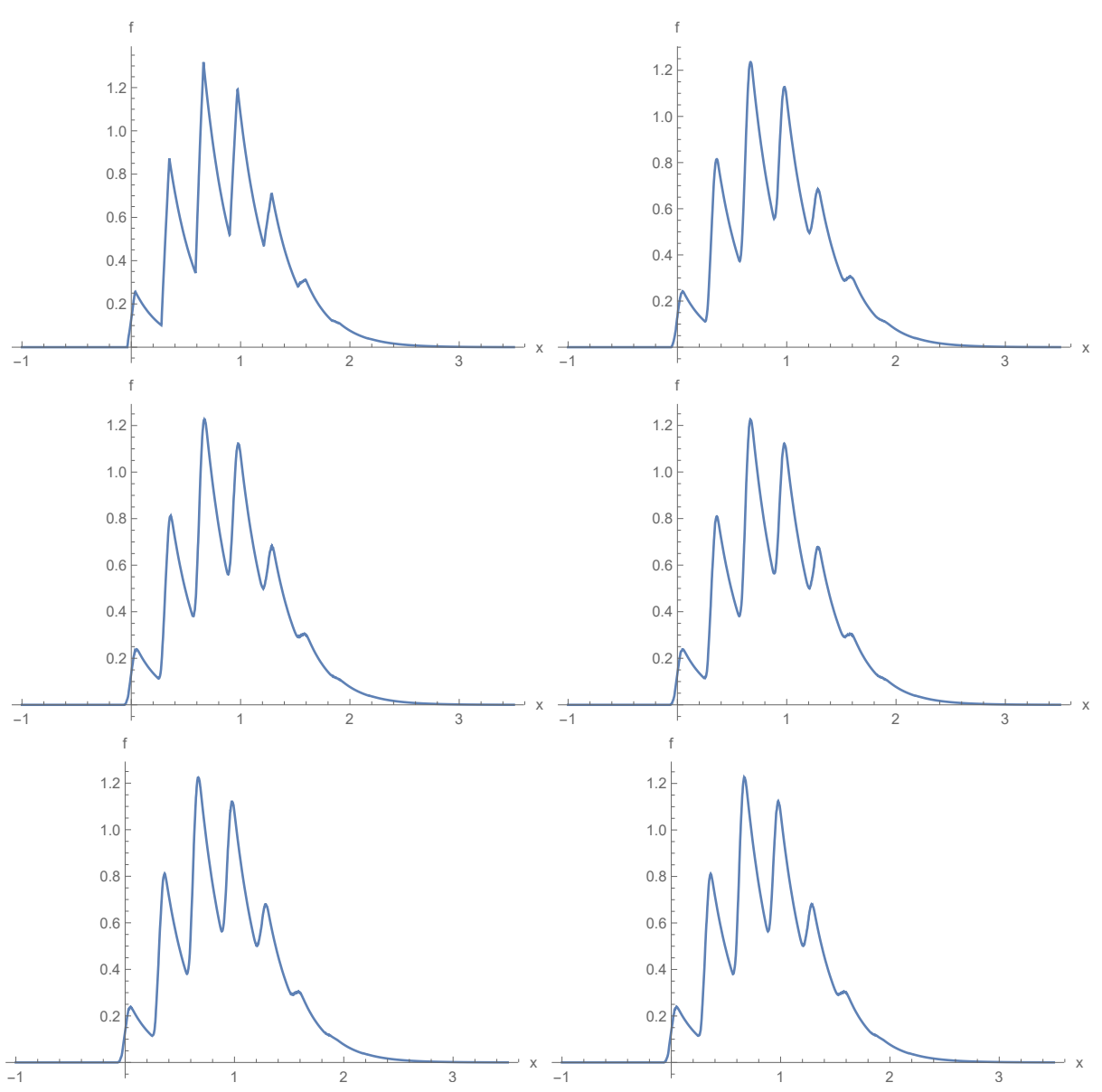

Figure 6. Density function $f_{X_{N}(0.5)}(x)$ for $N=1$ (up left), $N=2$ (up right), $N=3$ (center left), $N=4$ (center right), $N=5$ (down left) and $N=6$ (down right) in Example 3.5 .

for $t \in[0,1]$, where $A_{1}, A_{2}, \ldots$ are independent random variables with Poisson(5) distribution. Notice that the sum is well-defined in $\mathrm{L}^{2}([0,1] \times \Omega)$ : indeed,

$$
\left\|\frac{A_{n}}{n} t^{n}\right\|_{\mathrm{L}^{2}([0,1] \times \Omega)}=\frac{C}{n \sqrt{2 n+1}},
$$

where $C$ is the 2-norm of a Poisson(5) random variable, therefore

$$
\sum_{n=1}^{\infty}\left\|\frac{A_{n}}{n} t^{n}\right\|_{L^{2}([0,1] \times \Omega)}<\infty .
$$

It is assumed that $X_{0}, X_{1}, A_{1}, A_{2}, \ldots$ are independent.

By Theorem 2.5. $\lim _{N \rightarrow \infty} f_{X_{N}(t)}(x)=f_{X(t)}(x)$, for $t \in(0,1]$ and $x \in \mathbb{R}$. In Figure 8. we plot $f_{X_{N}(0.3)}(x)$ for $N=1,2,3,4,5,6$. We observe convergence, since virtually the same density functions are plotted at different values of truncation order $N$. 

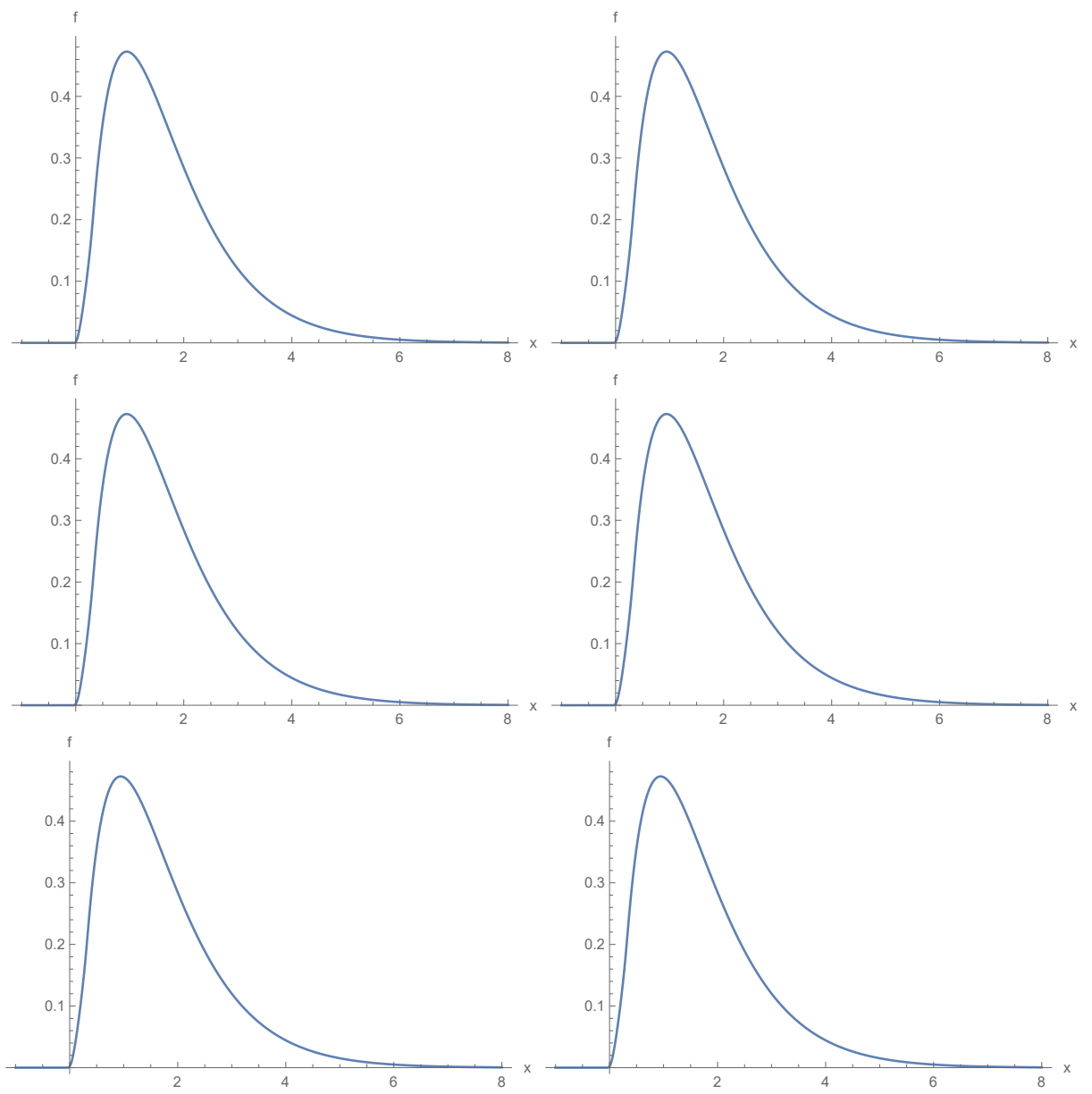

Figure 7. Density function $f_{X_{N}(0.5)}(x)$ for $N=1$ (up left), $N=2$ (up right), $N=3$ (center left), $N=4$ (center right), $N=5$ (down left) and $N=6$ (down right) in Example 3.6.

\section{Conclusions}

In this paper we have provided a comprehensive probabilistic analysis of the damped pendulum differential equation in the case that the initial conditions (position, $X_{0}$, and velocity, $\left.X_{1}\right)$ are random variables and the forcing term, $Y(t)$, is a stochastic process. To the best of our knowledge, a major difference of our contribution with respect to the ones available in the extant literature is that we have provided exact or approximate expressions for the probability density function of the solution stochastic process of this important problem in Physics. Our achievement contrasts with other studies, where the goal is merely to construct exact or approximate expressions for the mean and the variance of the solution process. We think that a strong point of our contribution is the wide range of scenarios studied with respect to the forcing term $Y(t)$ as well as the generality of our analysis. In particular, the important cases where $Y(t)$ is Gaussian (including the White noise process) or $Y(t)$ can be represented via Karhunen-Loève expansion or via a mean square convergent random power series have been fully addressed. Furthermore, 


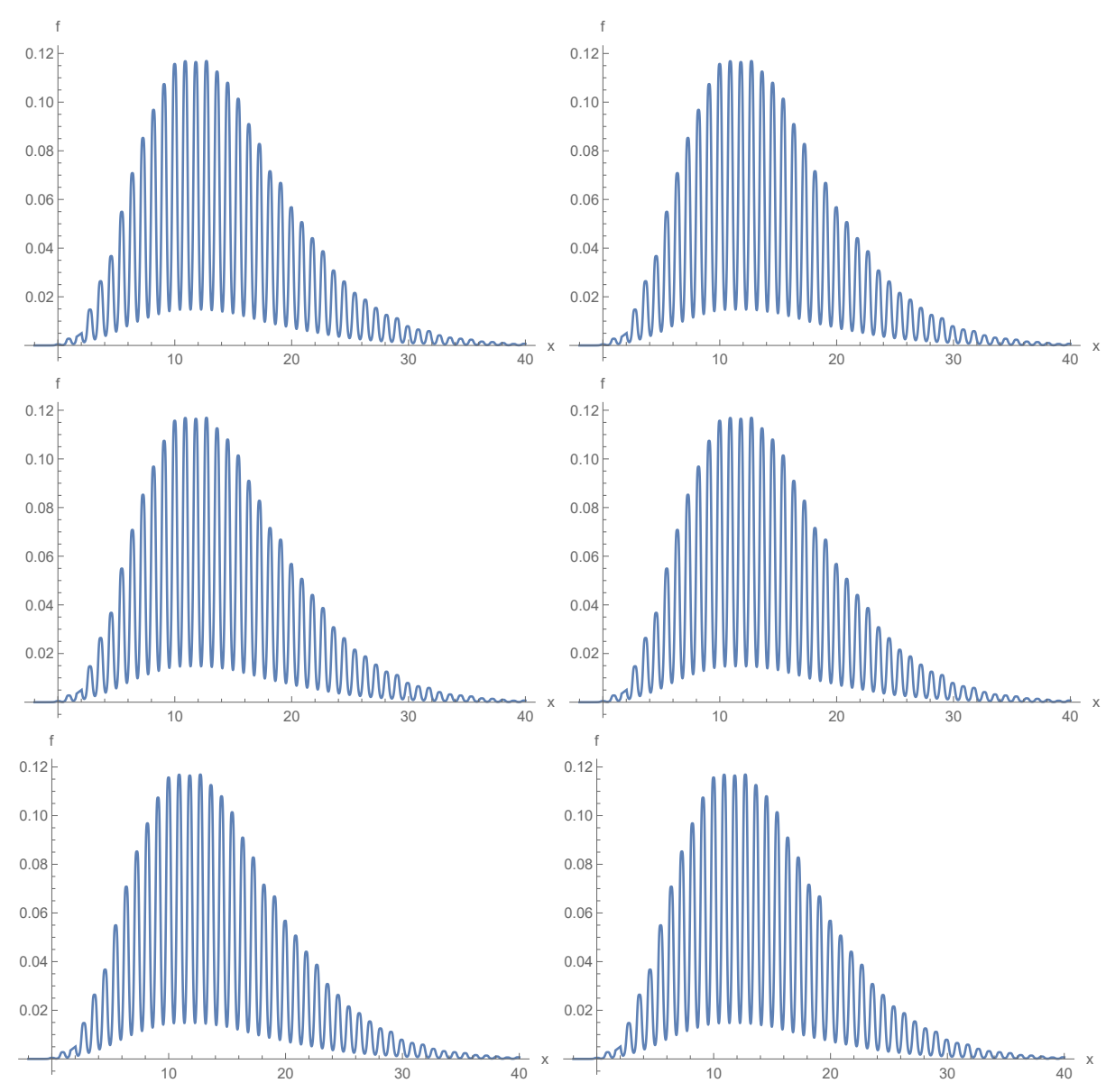

FiguRE 8. Density function $f_{X_{N}(0.3)}(x)$ for $N=1$ (up left), $N=2$ (up right), $N=3$ (center left), $N=4$ (center right), $N=5$ (down left) and $N=6$ (down right) in Example 3.7 .

the study has included a detailed discussion with regard to the hypotheses assumed on the input data $\left(X_{0}, X_{1}, Y(t)\right)$ to establish our findings. The generality of such hypotheses in practical situations has been illustrated throughout a wide variety of numerical examples. The analysis performed throughout this paper may be very useful in dealing with other random differential equations in future contributions.

\section{ACKNOWLEDGEMENTS}

This work has been supported by the Spanish Ministerio de Economía y Competitividad grant MTM2017-89664-P. Marc Jornet acknowledges the doctorate scholarship granted by Programa de Ayudas de Investigación y Desarrollo (PAID), Universitat Politècnica de València. The authors are grateful for the valuable comments raised by the reviewers that have improved the final version of the paper.

\section{Conflict of Interest Statement}

The authors declare that there is no conflict of interests regarding the publication of this article. 


\section{REFERENCES}

[1] Ralph C. Smith. Uncertainty Quantification. Theory, Implementation, and Application. SIAM Computational Science \& Engineering, 2014. ISBN: 9781611973211.

[2] A. T. Bharucha-Reid. On the theory of random equations. Proc. Sympos. Appl. Math., Vol. 16, 40-69, 1964, Amer. Math. Soc., Providence, R.I.

[3] T.T. Soong. Random Differential Equations in Science and Engineering. Academic Press, New York, 1973. ISBN: 9780080956121.

[4] M.H. Heydari, M.R. Hooshmandasl, C. Cattani and F.M. Maalek Ghainiab. An efficient computational method for solving nonlinear stochastic Itô integral equations: Application for stochastic problems in physics. Journal of Computational Physics, Vol. 283, 148-168, 2015. Doi: $10.1016 /$ j.jcp.2014.11.042.

[5] K. MalLick. Random oscillator with general Gaussian noise. Physica A: Statistical Mechanics and its Applications, Vol. 384(1), 64-68, 2007. Doi: 10.1016/j.physa.2007.04.070.

[6] F.A. Dorini and M. Cristina C. Cunha. Statistical moments of the random linear transport equation. Journal of Computational Physics 227(19) (2008) 8541-8550. doi:10.1016/j.jcp.2008.06.002.

[7] L.T. Santos, F.A. Dorini and M.C.C. Cunha. The probability density function to the random linear transport equation. Applied Mathematics and Computation 216(5) (2010) 15241530. doi:10.1016/10.1016/j.amc.2010.03.001.

[8] F.A. Dorini, F. Furtado and M. Cristina C. Cunha. On the evaluation of moments for solute transport by random velocity fields. Applied Numerical Mathematics 59(12) (2009) 2994-2998. doi:10.1016/j.apnum.2009.07.006.

[9] F.A. Dorini and M.C.C. CUnHA. On the linear advection equation subject to random velocity fields. Mathematics and Computers in Simulation 82(4) (2011) 679-690. doi:10.1016/j.matcom.2011.10.008.

[10] A. Hussein and M.M. Selim. Solution of the stochastic radiative transfer equation with Rayleigh scattering using RVT technique. Applied Mathematics and Computation 218(13) (2012) 7193-7203. doi:10.1016/j.amc.2011.12.088.

[11] A. Hussein and M.M. Selim. Solution of the stochastic generalized shallow-water wave equation using RVT technique. European Physical Journal Plus (2015) 130:249. doi:10.1140/epjp/i2015-15249-3.

[12] A. Hussein and M.M. SELIM. A general analytical solution for the stochastic Milne problem using Karhunen-Loève (K-L) expansion. Journal of Quantitative Spectroscopy and Radiative Transfer 125 (2013) 84-92. doi:10.1016/j.jqsrt.2013.03.018.

[13] G. Falsone and D. SetTineri. On the application of the probability transformation method for the analysis of discretized structures with uncertain proprieties. Probabilistic Engineering Mechanics 35 (2014) 44-51. doi: j.probengmech.2013.10.001.

[14] Zhijie Xu, Ramakrishna Tipireddy and Guang Lin. Analytical approximation and numerical studies of one-dimensional elliptic equation with random coefficients. Applied Mathematical Modelling 40(9-10) (2016) 5542-5559. doi:10.1016/j.apm.2015.12.041.

[15] J.L. Strand. Random ordinary differential equations. Journal of Differential Equations 7, 538-553 (1970). doi: 10.1016/0022-0396(70)90100-2.

[16] J.K. HaLe. Ordinary Differential Equations. Malabar: Robert E. Krieger Publishing Company, Second edition, 1980. ISBN: 9780486472119.

[17] P. Billingsley. Probability and Measure. John Wiley \& Sons, third edition, 1995. ISBN: 9781118122372.

[18] Gabriel J. Lord, Catherine E. Powell and Tony Shardlow. An Introduction to Computational Stochastic PDEs. Cambridge Texts in Applied Mathematics, Cambridge University Press, New York, 2014. ISBN: 9780521728522.

[19] J. Calatayud, J.-C. Cortés and M. JoRnet. On the approximation of the probability density function of the randomized heat equation. https://arxiv.org/pdf/1802.04190.pdf.

[20] Haim Brezis. Functional Analysis, Sobolev Spaces and Partial Differential Equations. Springer, 2011. ISBN: 9780387709130. 
[21] Walter Rudin. Principles of Mathematical Analysis. International Series in Pure \& Applied Mathematics, third edition, 1976. ISBN: 9780070542358.

[22] Antonio Ambrosetti and Giovanni Prodi. A Primer of Nonlinear Analysis. Cambridge Studies in Advanced Mathematics, 1993. ISBN: 9780521485739.

[23] M. M. VAinberg. Variational Methods for the Study of Nonlinear Operators. Holden-Day, 1964.

[24] A. W. van Der VaArt. Asymptotic Statistics. Cambridge University Press, 1998. ISBN: 9780521784504.

[25] Norbert Wiener and Aurel Wintner. Fourier-Stieltjes Transforms and Singular Infinite Convolutions. American Journal of Mathematics, Vol. 60, No. 3 (1938), pp. 513-522.

[26] Johnson N.L., Kotz S., Balakrishnan N. (1994). Continuous Univariate Distributions, Volume 1, Wiley.

[27] Bogdanoff J. L., Goldberg J. E., Bernard M. C. (1961). Response of a simple structure to a random earthquake-type disturbance. Bulletin of the Seismological Society of America, 51 (2), 293-310.

[28] Goldberg J. E., Bogdanoff J. L., Sharpe D. R. (1964). The response of simple nonlinear systems to a random disturbance of the earthquake type. Bulletin of the Seismological Society of America, 54 (1), 263-276.

[29] Kliemann W. (2018). Nonlinear Dynamics and Stochastic Mechanics. CRC Press.

[30] Maymon G. (1998). Some Engineering Applications in Random Vibrations $\& 3$ Random Structures. (Vol. 178). Aiaa.

[31] Caughey T. K. (1986). On the response of non-linear oscillators to stochastic excitation. Probabilistic Engineering Mechanics, 1 (1), 2-4.

[32] Allan Gut. Probability: A Graduate Course. Springer, 2005. ISBN: 9780387228334. 